\title{
Noncontact AFM and differential reflectance spectroscopy joint analyses of bis-pyrenyl thin films on bulk insulators: Relationship between structural and optical properties
}

\author{
Franck Bocquet, ${ }^{1}$ Laurent Nony, ${ }^{1, *}$ Franck Para, ${ }^{1}$ Philipda Luangprasert, ${ }^{1}$ Jean-Valère Naubron, ${ }^{2}$ Christian Loppacher, ${ }^{1}$ \\ Thomas Leoni, ${ }^{3}$ Anthony Thomas, ${ }^{3}$ Alain Ranguis, ${ }^{3}$ Anthony d'Aléo, ${ }^{3}$ Frédéric Fages, ${ }^{3}$ and Conrad Becker ${ }^{3}$ \\ ${ }^{1}$ Aix Marseille University, CNRS, IM2NP, UMR 7334, Marseille, France \\ ${ }^{2}$ Aix Marseille University, CNRS, Centrale Marseille, FR1739, Marseille, France \\ ${ }^{3}$ Aix Marseille University, CNRS, CINaM, UMR 7325, Marseille, France
}

(Received 2 March 2018; revised manuscript received 8 June 2018; published 22 June 2018)

\begin{abstract}
The combined characterization of structural and optical properties of organic interfaces adsorbed on inorganic solid substrates down to the molecular scale is crucial from a fundamental point of view, but also if one tackles efficient applied devices. In this work, a set of joint structural and optical analyses of self-assemblies of $\pi$ conjugated bis-pyrene derivatives upon adsorption on two alkali halides bulk insulators is reported. The structural analysis is performed by means of noncontact atomic force microscopy in ultrahigh vacuum either at room or at the liquid-nitrogen temperature with molecular resolution. The surface coverage ranges from the submonolayer (ML) regime up to 5 ML. In situ optical spectroscopy is performed by means of differential reflectance (DR) spectroscopy. A thorough fitting methodology of the DR spectra allows us to derive the complete dielectric function of the molecular adlayers treated in an anisotropic formalism, albeit restricted to an uniaxial approximation. Conclusions regarding the process of condensation of the molecules into $\mathrm{H}$ aggregates from its early stages up to the solid molecular phase are drawn. This work highlights three main reasons to bridge high-resolution structural and optical characterization of the molecular layers, which all point towards the necessity to constrain the fitting process, namely, (i) characterizing the growth mode of the molecules, (ii) identifying the structural order of the resulting assemblies, and (iii) discriminating their constitutive phases by means of molecular resolution imaging.
\end{abstract}

DOI: 10.1103/PhysRevB.97.235434

\section{INTRODUCTION}

To meet the demand of increased performances (efficiency, reliability, energy consumption, etc.), organic optoelectronic devices, among which are organic light-emitting diodes [1,2], organic solar cells [3-5], and organic field effect transistors [6,7], must rely on materials whose structure, adsorption, and absorption properties on solid surfaces must be tailored at best down to the nanoscale [8-12]. Among organic materials, $\pi$-conjugated materials are of particular importance because synthetic chemistry can provide molecular tectons with tunable optical and electronic properties along with a relative ease of production [5].

As mentioned, the functionality (optical and electronic properties) of these ultrathin organic-film-based devices relies on the structural properties of the interfacial molecular layers condensed upon adsorption on the substrate [8,11-17]. Therefore exploring the structure-property relations in these layers is necessary. The concept of interface layer meant here gathers organic-organic, or organic-inorganic interfaces, and encompasses coverage rates ranging from the submonolayer regime, the monolayer (ML) regime, up to the multilayer regime $(\simeq 1-10 \mathrm{ML})$. The thick-film regime $(>10 \mathrm{ML})$ is more particular because in this case, the resulting properties are representative of those of the bulk molecular crystal. Conversely,

\footnotetext{
*Author to whom correspondence should be addressed: laurent.nony@im2np.fr
}

in the multilayer regime, vertical (molecules-substrate) and lateral (intermolecular) interactions steer the resulting molecular growth mode along with the structure of the molecular arrangements. It is therefore crucial to characterize the structure of the interfacial layer for understanding its properties.

If optical properties are targeted, one must further make sure that the molecular assemblies develop well-defined electronic bands for proper light absorption, while keeping molecular electronic states which remain decoupled from those of the substrate. These criteria can be fulfilled when the molecules condense into ordered assemblies on dielectric substrates while retaining well-defined vertical and lateral interactions, as well as a weak charge transfer with the substrate to avoid optical quenching [18].

The characterization of these interfaces from a structural and an optical point of view down to the molecular scale is challenging and requires analytical methods with high sensitivity in an ultrahigh-vacuum (UHV) environment. On the one hand, scanning probe techniques, such as atomic force microscopy (AFM) in noncontact mode (nc-AFM), can provide access to the structure of the adsorbed molecular layers down to the molecular scale [19-23] and beyond [24-29]. The nc-AFM method, particularly on bulk insulators and at room temperature is demanding. However, as soon as the tip-surface interaction is stabilized, the resulting images give access to the growth mode and to the structure of the molecular arrangements quite straightforwardly. However, the information is local and not necessarily statistically representative of the structure of the molecules anywhere else on the substrate. On 


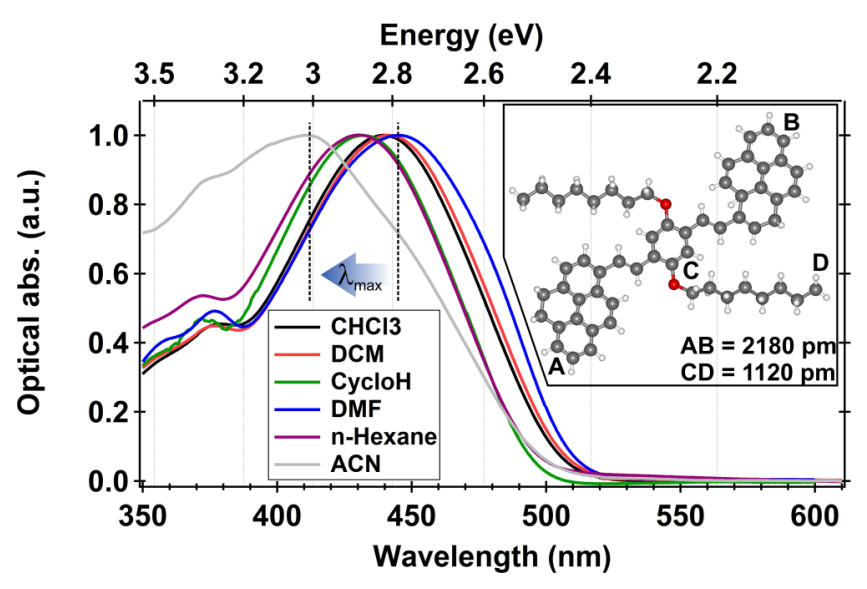

FIG. 1. Normalized absorption spectra of bis-pyrene molecules in various solvents. (Inset) The chemical structure of a bis-pyrene molecule with its dimensions.

the other hand, in situ differential reflectance spectroscopy (DRS) permits to measure the UV-vis absorption spectrum of the molecules with a sensitivity, including anisotropic sensitivity, to a fraction of a ML [30-33]. The spotlight size being macroscopic, the resulting differential reflectance (DR) spectrum is representative of a statistically relevant amount of molecules. However, because of its spectroscopic character, the interpretation of the resulting spectra is tedious. Important theoretical efforts are needed to extract relevant information out of the shifts, or the intensities of the peaks (influence of the vertical and lateral interactions in the layers, molecules orientation, transition dipole moments, excitonic effects, etc.). Therefore both methods are complementary. Regarding $\pi$ conjugated materials, it has been shown that the insertion of pyrene groups into conjugated oligomers yields highly conjugated structures with unique photophysical properties $[9,10,34,35]$.

In this work, we report joint nc-AFM and DRS analyzes of bis-pyrene thin films adsorbed on single crystals of alkali halides, namely, $\mathrm{KCl}(001)$ and $\mathrm{NaCl}(001)$. The combination between high-resolution imaging in real space and UV-visible absorption spectroscopy allows us to draw conclusions about the relationship between structural and optical properties of the molecular layers from the submonolayer regime up to five monolayers. The paper is organized as follows. In Sec. II, bis-pyrene molecules and alkali halide substrates are introduced along with the imaging and optical methods used to investigate them. In Sec. III, absorption and fluorescence spectra of the molecules in solvents are presented with both, the nc-AFM structural imaging of the molecules on $\mathrm{KCl}(001)$ and $\mathrm{NaCl}(001)$ from 0 to $5 \mathrm{ML}$, and their corresponding DR spectra. The results are combined and discussed in Sec. IV.

\section{MATERIALS AND METHODS}

\section{A. Bis-pyrene molecules and substrates}

1,4-Di-n-octyloxy-2,5-bis(pyren-1-ylethenyl)benzene (bispyrene) molecule is reported in Fig. 1 (inset). It is a $\pi$ conjugated, centrosymmetric, organic compound with pyrene groups connected to the central benzene group by $\sigma$ and double bonds. From pyrene to pyrene, the length of the molecule reaches $\simeq 2180 \mathrm{pm}$. The length of each alkyl chain is $\simeq 1100 \mathrm{pm}$. The alkyl chains are connected to the benzene by oxygen atoms, whose Lewis basic character should favor the adsorption on the cationic sites of our alkali halide substrates $[23,36]$. The structure of the molecule makes it weakly rigid with an ability to adapt its conformation (angle of tilt between the central benzene and the pyrene groups) to optimize its adsorption. Its structural arrangement and optical properties on gold has been reported in Refs. [37,38].

$\mathrm{NaCl}(001)$ and $\mathrm{KCl}(001)$ single crystals (MaTecK GmbH, 52428 Jülich, Germany) were cleaved ex situ with a resulting sample thickness $\gtrsim 1 \mathrm{~mm}$ and mechanically fixed on a sample holder. The backside interface between the substrate and the holder was beforehand intentionally made rough by micrometric mechanical scratching. The as-mounted sample is then quickly introduced into UHV, and annealed to $240^{\circ} \mathrm{C}$ during $1 \mathrm{~h}$ in order to obtain atomically clean surfaces with large terraces. Bis-pyrene molecules were deposited in situ onto the substrates kept at room temperature by thermo-evaporation from quartz crucibles at evaporation rates of $\simeq 0.3 \mathrm{ML} / \mathrm{min}$. A quartz crystal microbalance (QCMB) connected to a counter (TTi, TF930) and a homemade LabVIEW program allows for both, the control and the monitoring of the evaporation rate of the molecules. The crosscheck between the evaporation rate, the exposure time of the beam on the substrate and the nc-AFM images, allows for an estimation of the coverage rate of the molecules on the surface with an accuracy of $20 \%$.

\section{B. Absorption and fluorescence spectroscopy}

Electronic absorption spectra were recorded with a Jasco V670 instrument equipped with a peltier cell holder ETCS761 to maintain the temperature at $25.0^{\circ} \mathrm{C}$. A quartz cell with a 10-mm optical path length was used. Solutions of bis-pyrene with concentrations of 1,2 , and $4 \mathrm{mg} / 1(1.27 \times$ $10^{-6}, 2.54 \times 10^{-6}$, and $5.08 \times 10^{-6} \mathrm{~mol} / 1$, respectively) were prepared in N-hexane, cyclohexane, dichloromethane (DCM), N,N-dimethylformamide (DMF), chloroform and acetonitrile (ACN) (HPLC grade). Except with ACN, the molecules remain soluble and yield a yellowish solution. The absorption increases linearly with the concentration following a BeerLambert behavior. Conversely, with ACN, even at the lowest concentration investigated, the molecules are hardly soluble and rather yield macroscopic aggregates visible in the test tube. The UV-vis absorption spectra were recorded using the solvent as reference and are presented without further data processing. The measurement range was 300-800 $\mathrm{nm}$ with data interval of $1 \mathrm{~nm}$, a scan speed of $200 \mathrm{~nm} / \mathrm{min}$ and an UV-vis bandwidth of $1 \mathrm{~nm}$.

Fluorescence emission spectra were measured from solutions of bis-pyrene with similar concentrations and similar solvents as for absorption spectra. The spectra were recorded with a Jasco FP8600 spectrofluorometer equipped with a peltier cell holder FDP-837 to maintain the temperature at $25.0^{\circ} \mathrm{C}$ and using an excitation wavelength of $300 \mathrm{~nm}$. A quartz cell with a $10 \mathrm{~mm}$ optical path length was used. The measurement range was $300-700 \mathrm{~nm}$ with data interval of $1 \mathrm{~nm}$, scan speed of $100 \mathrm{~nm} / \mathrm{min}$, excitation and emission bandwidth of 5 and $2 \mathrm{~nm}$, respectively. 


\section{Noncontact atomic force microscopy}

Noncontact AFM images were either acquired at liquid nitrogen temperature $(\mathrm{LN} 2, \simeq 77 \mathrm{~K}$, temperature of the substrate, the tip is not cooled down), or at room temperature (RT) using a commercial AFM (VT-AFM, Omicron Nanotechnology $\mathrm{GmbH}, 65232$ Taunusstein, Germany) equipped with a homebuilt detection electronics and a R9 RHK controller (RHK Technology Inc., Troy, MI 48083, USA). Kelvin probe force microscopy was engaged during the acquisition of ncAFM images to compensate electrostatic forces and yield consistent contact potential difference (CPD) images. CPD images were performed in FM-KPFM mode with a $2345-\mathrm{Hz}$ modulation frequency detected on the side bands of the central frequency of the cantilever. The ac bias modulation was $1 \mathrm{~V}$. Nanosensor cantilevers were used (PPP-NCL with resonance frequency $f_{0}=157.760 \mathrm{kHz}$, spring constant $k_{0} \simeq 30 \mathrm{~N} / \mathrm{m}$, quality factor $Q=40000$ ) at oscillation amplitudes $A_{0} \simeq 2$ $\mathrm{nm}$ and frequency shifts $\Delta f \simeq-20 \mathrm{~Hz}$, hence a normalized $\Delta f$ [39]: $\gamma=k_{0} A_{0}^{3 / 2} \Delta f / f_{0} \simeq-0.01 \mathrm{nN} \sqrt{\mathrm{nm}}$. The estimated deposited thickness and the temperature at which each image was acquired (RT or LN2) is indicated in the top right corner of each figure.

Experimental nc-AFM images were analyzed using the wSXM software [40]. The topographical images which are presented here are raw data, only processed by a simple line-by-line flatten filter. Besides, a thorough set of postprocessing including cross-correlation, fast Fourier transform (FFT), histograms and corresponding Gaussian fits, allows us to quantify our measurements with typical $5 \%$ uncertainty. The data have been acquired and repeated over several weeks with various samples and tips. The reported images are the most representative of our results whose statistics is robust.

\section{Differential reflectance spectroscopy}

Differential reflectance spectroscopy (DRS) is a nondestructive, UV-visible-near IR spectroscopic method able to measure in real time and with a high sensitivity the change in reflectance on a substrate. In ultrahigh vacuum (UHV, base pressure $\simeq 10^{-10} \mathrm{mbar}$ ), the adsorbates (heteroatoms, molecules) can be thermally evaporated by sublimation on the surface of a substrate whereto they adsorb, thereby changing its reflectance. The DRS signal is defined as

$$
\operatorname{DRS}(d)=\frac{R(d)-R(0)}{R(0)},
$$

where $R(d)$ is the specular reflectance of the substrate with the deposited adlayer of thickness $d$ and $R(0)$ is the specular reflectance of the bare substrate. The differential aspect of the measure combined with the real time monitoring of the signal gives access to the changes of reflectance of the adsorbed layers, i.e., to their optical absorption properties, with a sensitivity to the submonolayer regime.

The DRS method has early been developed and rationalized out of the formalism of Fresnel's equations [30] for the case of semi-infinite, transparent, and nonabsorbing substrate, with the goal to extract the dielectric function of the adsorbed layer from its optical spectra [32,33]. In our case, both the roughness of the backside interface and the thickness of the substrate allows for neglecting the coherent back-reflection towards the front end of the sample and thus validates the semi-infinite assumption. Upon linearization of Fresnel's equations, the DRS signal with a semi-infinite and nonabsorbing substrate can be interpreted as scaling linearly with the imaginary part of the dielectric function of the adsorbed layer [30,32]. In that sense, it relates to the optical density, as measured by regular spectroscopic methods in solution [32]. This approximation is correct for layers (i) that can be assumed as continuous and forming an isotropic medium and (ii) whose thickness $d$ is much smaller than the wavelength of the incoming light.

The method now permits to perform polarization-sensitive measurements [41,42], with a sensitivity in the $10^{-4}$ range [43]. Concerning atomic adsorbates on substrates, DRS has been used to investigate the optical properties of oxygen and hydrogen on $\mathrm{Si}(001)$ [44-47], oxygen on $\operatorname{InAs}(001)$ [48], heteroatoms on III-V compounds [49], Si on $\mathrm{Ag}(110)$ [41], etc. to name a few. The adsorption of molecules on substrates was also intensively studied, among which is the exemplary case of perylene on mica(0001) [32,50,51], on $\mathrm{Au}(111)$ [32], on $\mathrm{KCl}(001)$ [51], on graphene, and hexagonal boron nitride [52], but as well, ethylene on $\mathrm{Si}(001)$ [53], phthalocyanine derivatives on $\mathrm{Au}(111)$ [54] and $\mathrm{Si}(001)-2 \times 1$ [55], perfluoropentacene on $\mathrm{Ag}(110)$ [42] and on $\mathrm{SiO}_{2}$ [56], pentacene on quartz (0001) [57] and on $\mathrm{SiO}_{2}$ [56], $\alpha$-sexithiophene on $\mathrm{Cu}(110)-(2 \times 1) \mathrm{O}$ [58], etc. Other results are reviewed in Refs. $[33,59,60]$.

Several reviews dealing with the DRS method have been published [32,33]. Therefore it will not be detailed further on here. Nevertheless, it is important to remind some of its main underlying mechanisms and what the specificities of our setup are. This is reported in Appendix. Briefly, our DRS setup is implemented in the preparation chamber of a UHV setup consisting of two interconnected chambers (base pressure $\simeq 10^{-10}$ mbar), as shown in Fig. 15. The molecules are sublimated from an evaporator stage (No. 1 in the figure) consisting of a quartz crucible mounted on a home made transferable holder. Unpolarized, white light is focused by means of a set of ex situ lenses towards the sample while the sublimation takes place. The reflected light is collected ex situ and then injected into a spectrometer allowing for the real-time monitoring of the DRS signal.

\section{RESULTS}

\section{A. Absorption and fluorescence spectroscopy of bis-pyrene in solvents}

\section{Absorption spectroscopy}

The UV-visible absorption properties of the bis-pyrene molecule were first measured in several aprotic solvents in order to avoid any specific solute-solvent interaction such as hydrogen bonding, namely, N-hexane, cyclohexane, dichloromethane (DCM), N,N-dimethylformamide (DMF), chloroform and acetonitrile (ACN). The properties of those solvents spread from weakly dispersive and apolar (N-hexane, cyclohexane) to strongly dispersive and polar (ACN and DMF), DCM and chloroform being intermediate. The relative dielectric permittivity $\left(\epsilon_{r} \simeq 37\right)$ and the dipole moment $(\simeq 3.9 \mathrm{D})$ of ACN and DMF are nearly similar. In apolar, aprotic, and weakly dispersive solvents, the spectra are expected to be more 


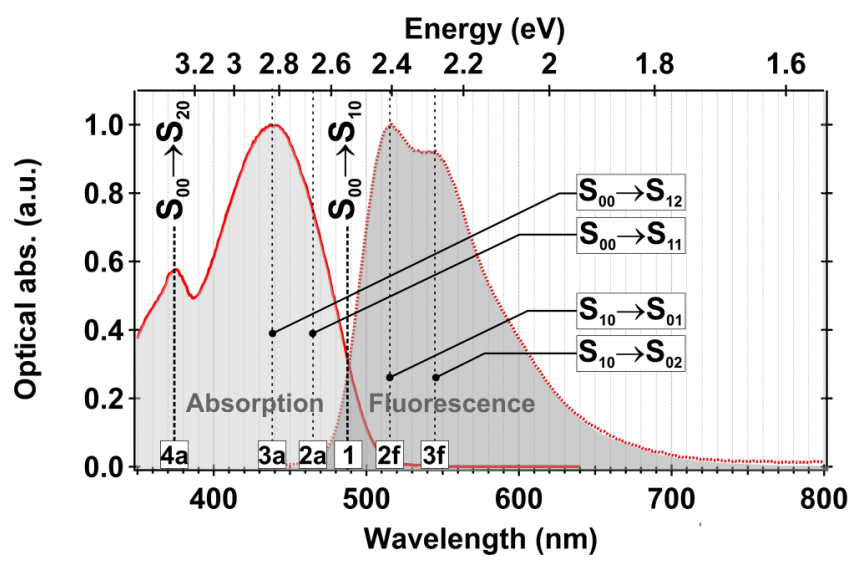

FIG. 2. Normalized absorption and fluorescence spectra of bispyrene in DCM. The excitation wavelength for fluorescence spectroscopy is $300 \mathrm{~nm}$.

representative of the absorption of molecules in a gaseous phase.

Normalized absorption spectra are reported in Fig. 1. The normalization is performed w.r.t. the maximum of the absorption intensity. The spectra are all measured with wellsolubilized molecules (except with $\mathrm{ACN}$ ) and are quite similar. They exhibit a large and featureless absorption peak at $(\simeq 440 \pm 2) \mathrm{nm}[(2.821 \pm 0.013) \mathrm{eV}]$ and a small feature at $(375 \pm 2) \mathrm{nm}[(3.310 \pm 0.018) \mathrm{eV}]$. These are representative of the $S_{0} \rightarrow S_{1}$ and of the $S_{0} \rightarrow S_{2}$ transitions, respectively [37]. A moderate solvatochromism $( \pm 7 \mathrm{~nm})$ at the absorption maximum is observed. Spectra in N-hexane and cyclohexane (apolar solvents) are superposed, which is expected because they have similar permittivities $\left(\epsilon_{r} \simeq 2\right)$. The ones obtained in DCM $\left(\epsilon_{r}=8.9\right.$, dipolar moment $\left.=1.5 \mathrm{D}\right)$ and chloroform $\left(\epsilon_{r}=5\right.$, dipolar moment $\left.=1.2 \mathrm{D}\right)$ are similar as well, but however exhibit a redshift with respect to (w.r.t) the cyclohexane absorption spectrum. The spectrum measured in DMF is the most redshifted. Except for ACN, considering the moderate solvatochromism and the stability of their shape, the experimental spectra shown in Fig. 1 are assumed to be representative of the response of an isolated molecule.

A detailed inspection of the spectra reveals that $40 \mathrm{~nm}$ higher than the maximum peak, a shoulder (which is more pronounced in DMF) is visible, which can be related to the reminiscence of a vibronic progression but overall, no welldeveloped vibronic replica are observed in the spectra. For a better interpretation of the optical fingerprints of a molecule, it is important to combine its absorption and fluorescence spectra.

\section{Fluorescence spectroscopy}

The fluorescence normalized spectrum of bis-pyrene in DCM is reported in Fig. 2, along with the corresponding absorption spectrum. For solubilized molecules (i.e., except in $\mathrm{ACN}$ ), fluorescence spectra look similar whatever the solvent used, consistently with the trend reported for the absorption spectra with a Stokes shift (energy gap between absorption and fluorescence maxima) of $\simeq(80 \pm 5) \mathrm{nm}$. We chose to report only the spectra in DCM because this behaves like an intermediate solvent from both polarity and permittivity point
TABLE I. Table of the absorption (label "a") and fluorescence (label " $\mathrm{f}$ ") peaks of bis-pyrene molecules in DCM.

\begin{tabular}{llccc}
\hline \hline & & $\lambda$ & $\begin{array}{c}\text { Energy } \\
(\mathrm{eV})\end{array}$ & $\begin{array}{c}\left(E_{x}-E_{0}\right) \\
(\mathrm{eV})\end{array}$ \\
\hline $4 \mathrm{a}$ & $S_{00} \rightarrow S_{20}$ & $(375 \pm 2)$ & $(3.310 \pm 0.018)$ & - \\
$3 \mathrm{a}$ & $S_{00} \rightarrow S_{12}$ & $(440 \pm 2)$ & $(2.821 \pm 0.013)$ & $(+0.277 \pm 0.023)$ \\
$2 a$ & $S_{00} \rightarrow S_{11}$ & $(463 \pm 2)$ & $(2.681 \pm 0.012)$ & $(+0.137 \pm 0.022)$ \\
1 & $S_{00} \rightarrow S_{10}$ & $(488 \pm 2)$ & $(2.544 \pm 0.010)$ & 0 \\
$2 \mathrm{f}$ & $S_{10} \rightarrow S_{01}$ & $(515 \pm 2)$ & $(2.410 \pm 0.009)$ & $(-0.133 \pm 0.019)$ \\
$3 \mathrm{f}$ & $S_{10} \rightarrow S_{02}$ & $(545 \pm 2)$ & $(2.278 \pm 0.008)$ & $(-0.266 \pm 0.018)$ \\
\hline \hline
\end{tabular}

of view. The location of the overlap between absorption and fluorescence spectra (axis labeled 1 in Fig. 2) remains nearly unchanged $(488 \pm 2) \mathrm{nm}\left[E_{1}=(2.544 \pm 0.010) \mathrm{eV}\right]$ for all the solvents used.

The so-called "mirror rule" [61] is violated around the axis 1. As stated by this rule in most cases [62-64] (i) the overlap stands for the $S_{00} \rightarrow S_{10}$ transition [the first (second) index gives the electronic (vibronic) level of the transition] and (ii) the Stokes shift is an integer number of vibrons (gap between two consecutive vibronic transitions). Here, the violation is particularly prominent judging from the well-developed peaks in the fluorescence spectrum (labelled $2 \mathrm{f}$ and $3 \mathrm{f}$ in Fig. 2), which are absent in the absorption counterpart. The symmetric transition to the $S_{0} \rightarrow S_{2}$ one is naturally absent in the fluorescence spectrum due to the radiation-less relaxation of the electronic excited state related to Kasha's rule, namely, that any fluorescent emission may only occur from a totally relaxed $\pi^{\star}$ excited state.

Considering both the small Stokes shift change, the constant energy position of the overlap and the weak solvatochromism reported in Fig. 1, it is inferred that, as explained in Ref. [65], the breakdown of the mirror rule stems from the flexibility of the molecular skeleton (called libration), which smears out the vibronic progression in absorption but not in fluorescence, as observed in Fig. 2. Following this, the fluorescence emission peaks can now be assigned in Fig. 2. The axis labelled 1 stands for the $S_{00} \rightarrow S_{10}$ vibronic transition: $\lambda_{1}=(488 \pm 2)$ $\mathrm{nm}, E_{1}=(2.544 \pm 0.010) \mathrm{eV}$. The axis labeled $2 \mathrm{f}$ stands for the second vibronic transition $S_{10} \rightarrow S_{01}: \lambda_{2}=(515 \pm$ 2) $\mathrm{nm}, E_{2}=(410 \pm 0.009) \mathrm{eV}$. The axis $3 \mathrm{f}$ stands for the third vibronic transition $S_{10} \rightarrow S_{02}: \lambda_{3}=(545 \pm 2) \mathrm{nm}, E_{3}=$ $(2.278 \pm 0.008) \mathrm{eV}$. These values are reported in Table I. In Fig. 2, the symmetric peaks to $2 \mathrm{f}$ and $3 \mathrm{f}$ ones w.r.t axis 1 are drawn in the absorption spectrum ( $2 \mathrm{a}$ and $3 \mathrm{a}$, respectively). They correspond to the vibronic progression during absorption, as they should appear if they were not smeared out by the libration phenomena. A lightweight shoulder had been noticed in the absorption spectra, notably in DMF, which had been interpreted in terms of smeared out vibronic replica. Consistently with the present interpretation, this shoulder is ascribed to the $S_{00} \rightarrow S_{11}$ vibronic transition.

\section{Optical properties upon aggregation in ACN}

In $\mathrm{ACN}$, bis-pyrene are poorly solubilized (aggregation in the test tube). The corresponding spectrum (cf. Fig. 1) is rather representative of an aggregated phase characterized by 
a blue-shift (hypsochromism effect) of the intensity maximum (transition occurring at $\lambda_{\max }$ ). This occurs along with a large reorganization of the intensity of the spectrum [cf. for instance the $\left(S_{0} \rightarrow S_{1}\right) /\left(S_{0} \rightarrow S_{2}\right)$ ratio]. This behavior is well-documented [66-71] and due to a packing of the molecules into so-called $\mathrm{H}$-aggregates. Owing to the molecular structure of bis-pyrene, the macroscopic aggregation of those molecules in ACN is expected to be locally driven by $\pi-\pi$ interactions [38].

\section{B. nc-AFM imaging of bis-pyrene on $\mathrm{KCl}(001)$}

The structural evolution of the growth of bis-pyrene on $\mathrm{KCl}(001)$ is now described. Three regimes are distinguished: the submonolayer regime ( 0 to $\simeq 0.5 \mathrm{ML}$ ), the ML regime $(0.5 \mathrm{ML}$ to $\simeq 1.5 \mathrm{ML})$, and the thick-layer regime ( $\simeq 5 \mathrm{ML})$.

\section{Submonolayer regime: 0 to $0.5 \mathrm{ML}$}

In the early stages of growth, the molecules form extended domains $\left(>200 \times 200 \mathrm{~nm}^{2}\right.$ in size) on the $\mathrm{KCl}$ terraces and decorate the step edges [cf. Figs. 3(a) and 3(b)]. The morphology of these domains seems to be limited by the distribution of step edges of the substrate [cf. Figs. 3(b) and 3(c)]. No domains were found on the terraces without step edges as boundaries. Therefore at room temperature, the molecules have a long diffusion length ( $\gtrsim 200 \mathrm{~nm})$ and do not nucleate on the terraces, meaning that their interaction with the substrate is weak. Except on step edges and on kink sites of the substrate, the corresponding CPD image to Fig. 3(c) [cf. Fig. 3(d)] presents a rather homogeneous contrast, with a mean value $\simeq-1 \mathrm{~V}$. The corresponding histogram is well-fitted with two Gaussian profiles only [cf. Fig. 3(f)]. The main one, $\simeq-1 \mathrm{~V}$, stands for the CPD mean value of the image. The second one, with a much smaller weight, reaches $-0.6 \mathrm{~V}$. It is assigned to the bright contrast seen at step edges and kink sites [cf. circles in Fig. 3(d)], whereas the latter is representative of the CPD on $\mathrm{KCl}$ terraces. The relative change between CPDs means that step edges and kink sites are negatively charged, a yet reported observation on alkali halide single crystals due to double layer effects [72,73]. Any relevant Gaussian profile can be unambiguously assigned to the CPD of the molecules. This means that in the areas of the molecular islands located far from step edges or kink sites, the adsorbed molecules neither carry net charges nor dipoles which are measurable with our setup. Therefore the corresponding height measurements are all the more reliable.

Figures 3(c) and 4(a) allow for an estimate of the apparent height of the molecular domains. In both figures, the topographical nc-AFM image presents two terraces separated by a monatomic step (height: $330 \mathrm{pm}$ ), similar to those seen in Fig. 3(a). The lower terrace is filled with a molecular domain. On both areas, the deduced apparent height of the domain is (780 \pm 40$)$ pm [cf. corresponding cross-sections in Figs. 3(e) and 4(b), respectively]. This molecular phase will be referred to as the " $\alpha$ " phase. For small ( $<50$ atoms) $\pi$-conjugated organic compounds adsorbed in a flat-lying configuration, this apparent height is large. Typical reported values give $\simeq 350 \mathrm{pm}[19,22]$.

According to the aspect ratio of the molecule, it is unlikely that the $\alpha$ phase consists of molecules standing vertically, with their long axis aligned perpendicularly w.r.t. the substrate
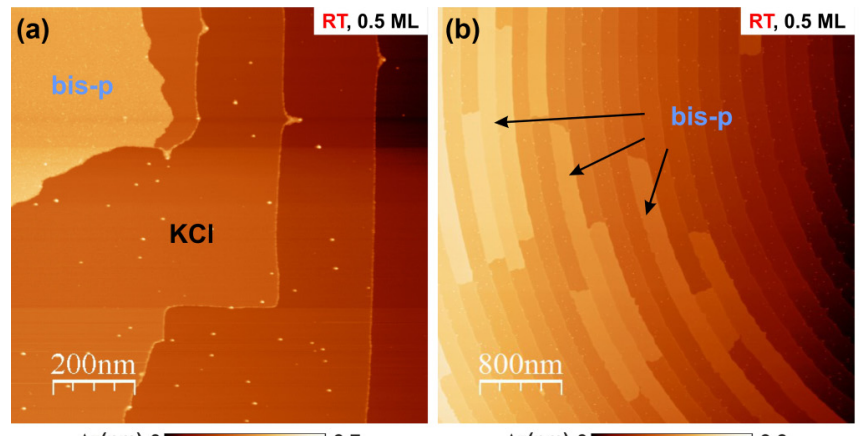
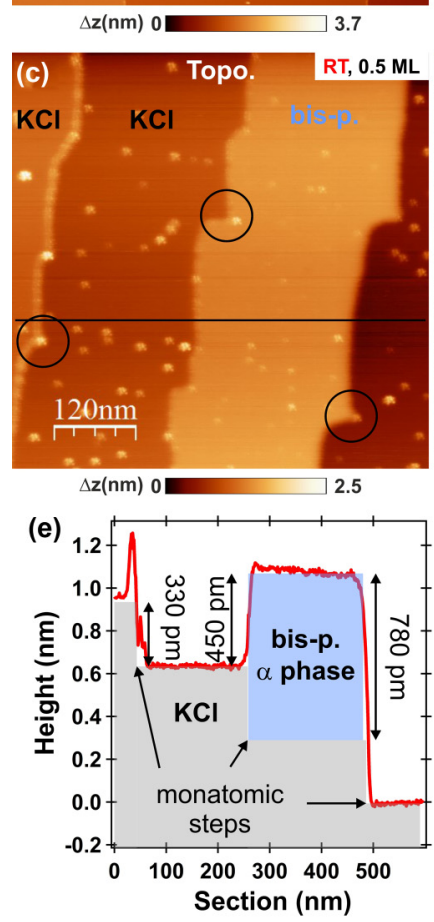

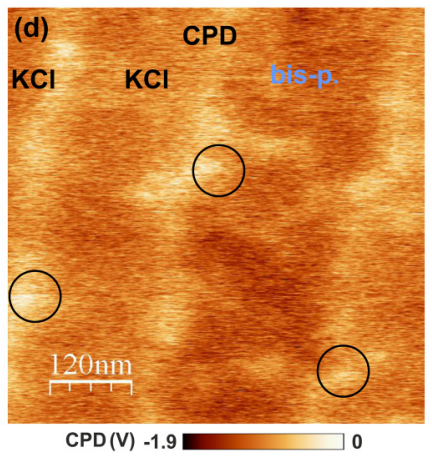

(f)

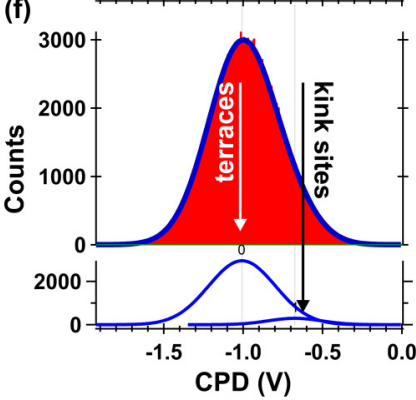

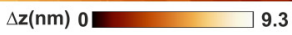

FIG. 3. (a)-(c) nc-AFM topographical images of $0.5 \mathrm{ML}$ bispyrene on $\mathrm{KCl}(001)$ taken on three samples [(a) $4 \times 4 \mu \mathrm{m}^{2}$, (b) $1 \times 1 \mu \mathrm{m}^{2}$, and (c) $600 \times 600 \mathrm{~nm}^{2}$ ]. (d) CPD image corresponding to (c). (e) Cross-section along the black line in (c). (f) Histogram of image (d) and corresponding Gaussian fits.

plane. They must rather be in a nearly flat-lying configuration. However, even in this situation, two cases can be envisioned: (1) the molecules may be adsorbed in a fully conjugated configuration with the planes of the pyrene groups aligned with the central phenyl ring, without, however, developing a strong interaction with the substrate (weakly corrugated van der Waals background). In this configuration, the molecules undergo a large in-plane diffusion amplified by the quick and random motion of the their alkyl chains (length $\simeq 1120 \mathrm{pm}$ ) due to thermal agitation. Indeed, molecular dynamic simulations on other molecules, but with similar side groups, have shown how mobile these parts are at room temperature [74]. This continuous motion of the alkyl chains not only increases the equivalent volume of sterical hindrance of each molecule, hence makes it appearing "high" upon imaging, but also prevents a side molecule from approaching close enough, or in an appropriate configuration, to develop a robust supramolecular interaction. This motion destabilizes their adsorption on the substrate too, which prevents them from finding a stable adsorption site. 

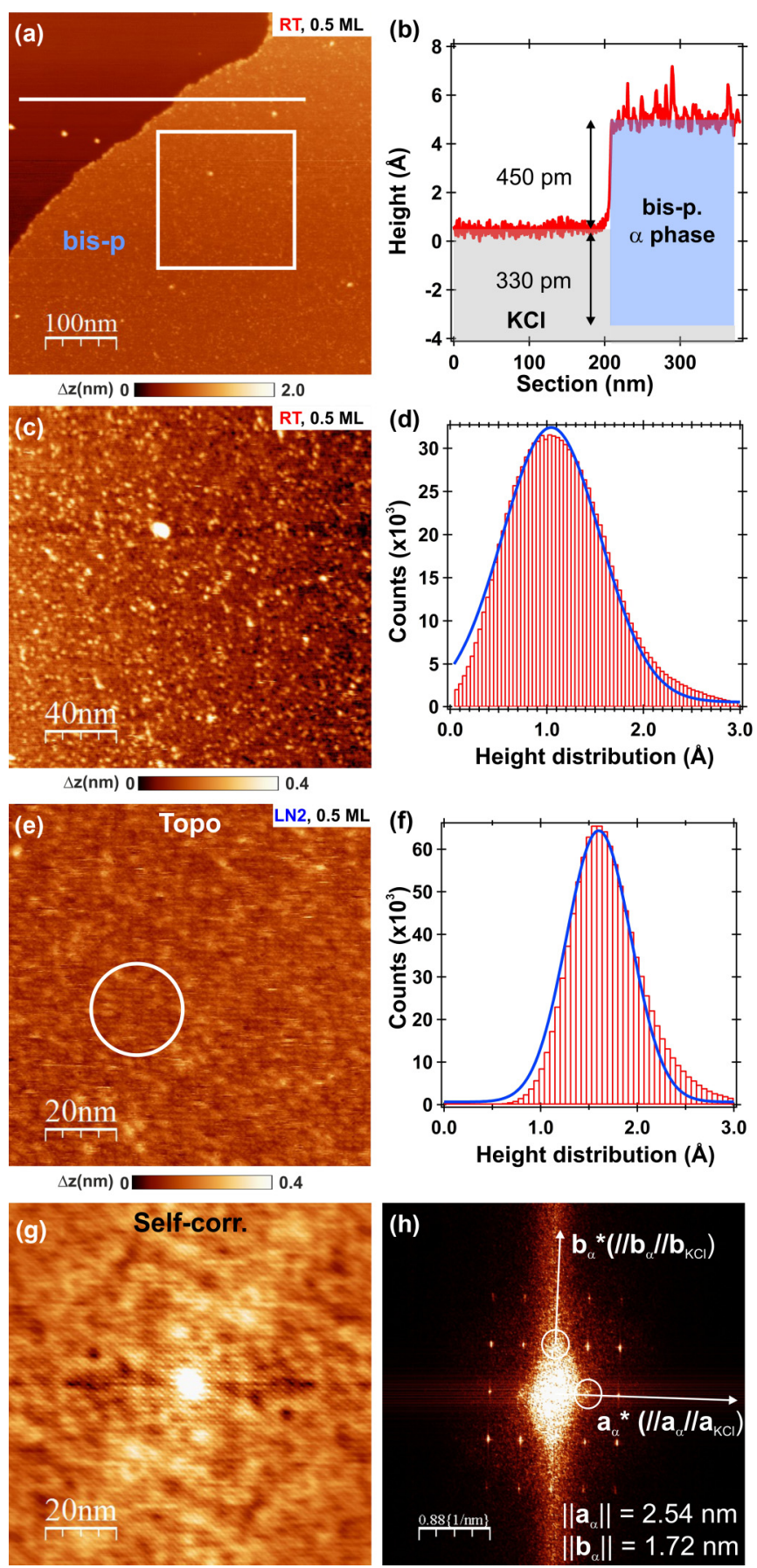

FIG. 4. (a) $500 \times 500 \mathrm{~nm}^{2}$ nc-AFM topographical image of $0.5 \mathrm{ML}$ bis-pyrene on $\mathrm{KCl}(001)$. The molecular assembly is visible in the lower $\mathrm{KCl}$ terrace (the right-hand side). (b) Cross-section corresponding to the white line in (a). (c) Zoom in corresponding to the squared area in (a). The structural disorder of the layer is visible. (d) Height distribution corresponding to image (c). A Gaussian profile has been fitted on top of it (thick blue curve) whose width is $(715 \pm 10) \mathrm{nm}$. (e) Similar sample, but imaged at liquid nitrogen temperature. $100 \times 100 \mathrm{~nm}^{2}$. Some parts (circle) exhibit regular molecular arrangements. (f) Height distribution corresponding to image (e). The Gaussian profile used for fitting the distribution (thick blue curve) gives a width of $(470 \pm 10) \mathrm{pm}$. (g) Self-correlated image of image (e). Periodic features are visible, which testifies to the regular arrangement of the molecules. (h )Modulus of the fast Fourier transform of image $(\mathrm{g})$.
(2) Each molecule is adsorbed in a configuration for which the $\pi$ conjugation is partly lost, i.e., in a configuration where the plane of the pyrene groups is tilted w.r.t. the plane of the central benzene. Then, the apparent height of the molecule must be increased and intermolecular $\pi-\pi$ stacking might be favored. This should favor the nucleation of the molecules into supramolecular domains. We do not see how the alkyl chains would be arranged in this configuration, but they might still contribute to the large apparent height of the molecules.

Despite several attempts, it was impossible to resolve the structure of the $\alpha$ phase with high resolution at room temperature [cf. Fig. 4(c)]. If the apparent height of the layer was confirmed over all the tested areas, the layer seems systematically rough. The histogram corresponding to Fig. 4(c) is shown in Fig. 4(d). It can be fitted with a Gaussian profile whose standard deviation is $\sigma=(360 \pm 10) \mathrm{pm}$. The former value relates to the layer roughness. In the case of a disordered ML on a substrate, the averaged layer height $h$ can thus be roughly extrapolated to $h \simeq 2 \sigma=(720 \pm 20) \mathrm{pm}$, in reasonable agreement with the apparent height of the layer derived from the step edge. The apparent disorder of the molecular layer and the impossibility for the tip to develop a local, stable, interaction with the molecules is consistent with assumption 1 discussed above.

Then the sample was cooled from RT down to liquid nitrogen temperature $(\simeq 77 \mathrm{~K})$ and the nc-AFM images acquired at that temperature. Note that the flow cryostat configuration of our VT-AFM allows for the cooling of the sample only, not of the tip. Figure 4(e) shows a typical image of the $\alpha$ phase acquired in these conditions. Highly-ordered areas can now be identified [cf. circle in Fig. 4(e)] with other areas where the contrast gets blurry. The apparent height of the layer at that temperature is $(520 \pm 20) \mathrm{pm}$. The histogram shown in Fig. 4(f) can be fitted with a Gaussian profile giving $h=(470 \pm 10) \mathrm{pm}$, which is consistent with the apparent height of the layer at that temperature. Therefore, compared to RT measurements, the roughness is significantly lowered at LN2 temperature. The self-correlated image of the former topographical image and its corresponding FFT [cf. Figs. 4(g) and 4(h), respectively] reveal a periodic structure for the $\alpha$ phase with a rectangular unit cell (u.c.) $\left\|\mathbf{a}_{\alpha}\right\|=(2.5 \pm 0.1)$ $\mathrm{nm},\left\|\mathbf{b}_{\alpha}\right\|=(1.7 \pm 0.1) \mathrm{nm}$, and $\left(\widehat{\mathbf{a}_{\alpha}, \mathbf{b}_{\alpha}}\right)=(90 \pm 2)^{\circ}$ including one molecule. The surface density for the $\alpha$ phase is $\simeq 0.24 \mathrm{molnm}^{-2}$. $\left\|\mathbf{a}_{\alpha}\right\|$ and $\left\|\mathbf{b}_{\alpha}\right\|$ axes are aligned along the crystallographic axes of the substrate $(\langle 100\rangle$ and $\langle 010\rangle$, respectively). This configuration points towards a higher-order commensurate epitaxy as a supercell can be identified [75]:

$$
\left(\begin{array}{l}
\mathbf{a}_{\alpha} \\
\mathbf{b}_{\alpha}
\end{array}\right)=\left(\begin{array}{cc}
4 & 0 \\
0 & 2.5
\end{array}\right)\left(\begin{array}{l}
\mathbf{a}_{\mathrm{KCl}} \\
\mathbf{b}_{\mathrm{KCl}}
\end{array}\right) .
$$

The comparison between the results acquired at RT and at LN2 allows us to reject assumption 2 because the molecules are too far apart to develop any $\pi$ stacking. Assumption 1 is rather to be considered. As sketched in Fig. 5, the low temperature lowers the molecular mobility observed at RT by lowering the motion of the alkyl chains of the molecules. This allows for both, the stabilization of the supramolecular phase by the development of an epitaxial relation with the substrate and the tip to come closer to the molecules while keeping an 

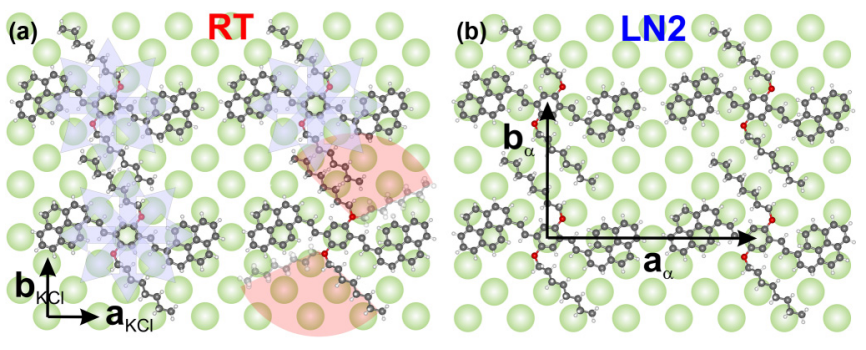

FIG. 5. Sketch of the $\alpha$-phase molecular arrangement at room (a) and $\mathrm{LN} 2$ (b) temperature on $\mathrm{KCl}(001)$. Only one type of ions of the substrate are drawn.

overall stable interaction, which ultimately permits to resolve the structure of the arrangement.

\section{Monolayer regime: 1 to $1.5 \mathrm{ML}$}

The morphology of the bis-pyrene film on $\mathrm{KCl}$ at $\mathrm{RT}$ for a coverage of $\simeq 1.5 \mathrm{ML}$ is reported in Fig. 6 . The molecules condense into large domains with irregular shapes $(>200 \times$
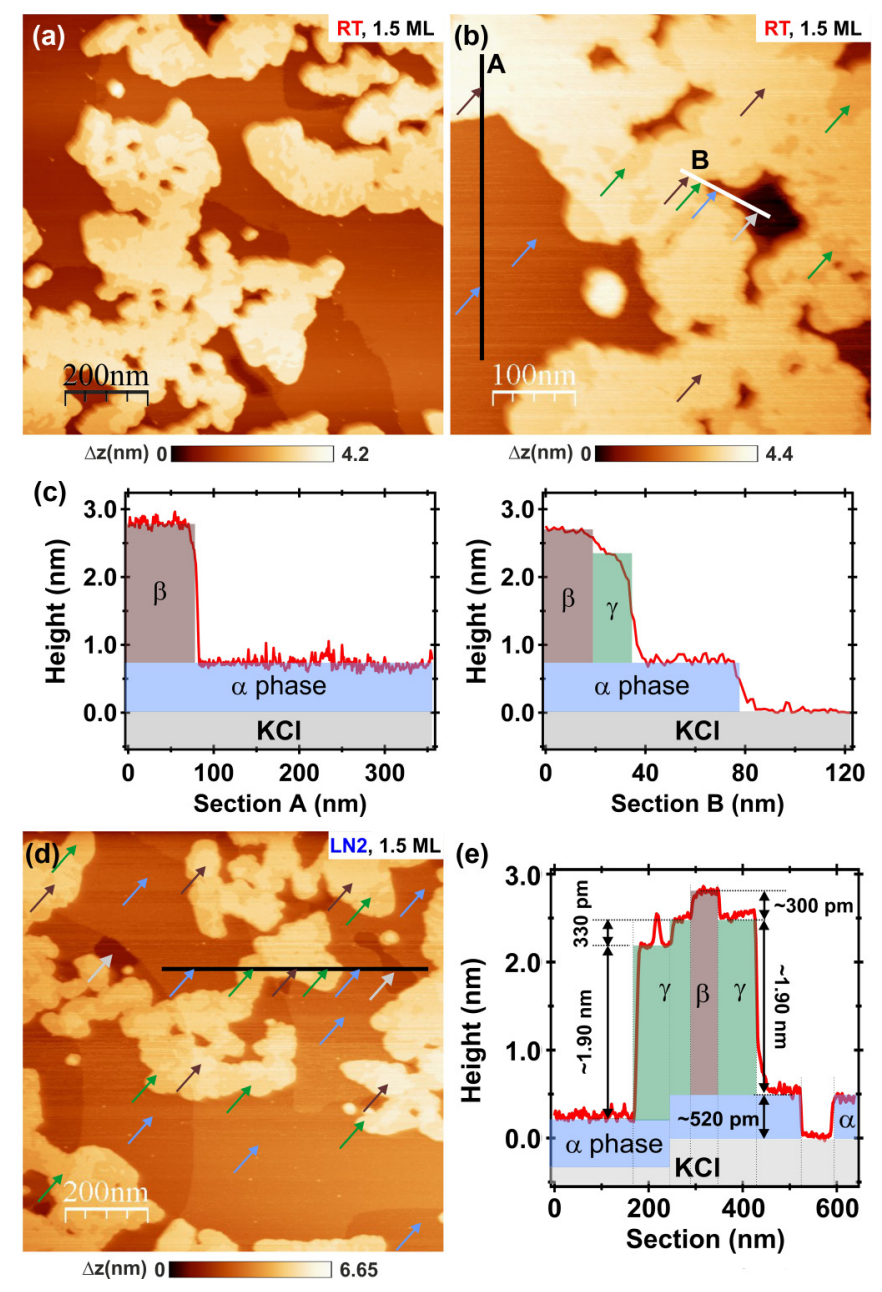

FIG. 6. [(a) and (b)] nc-AFM topographical images of 1.5 ML bispyrene on $\mathrm{KCl}(001)$ at room temperature $\left[\left(\right.\right.$ a) $1 \times 1 \mu \mathrm{m}^{2}$, (b) $500 \times$ $500 \mathrm{~nm}^{2}$ ]. (c) Height profiles corresponding to A and B cross sections visible in (b). (d) nc-AFM topographical image of 1.5 ML bis-pyrene on $\mathrm{KCl}(001)$ at $\mathrm{LN} 2$ temperature $\left(1 \times 1 \mu \mathrm{m}^{2}\right)$ and (e), corresponding profile.
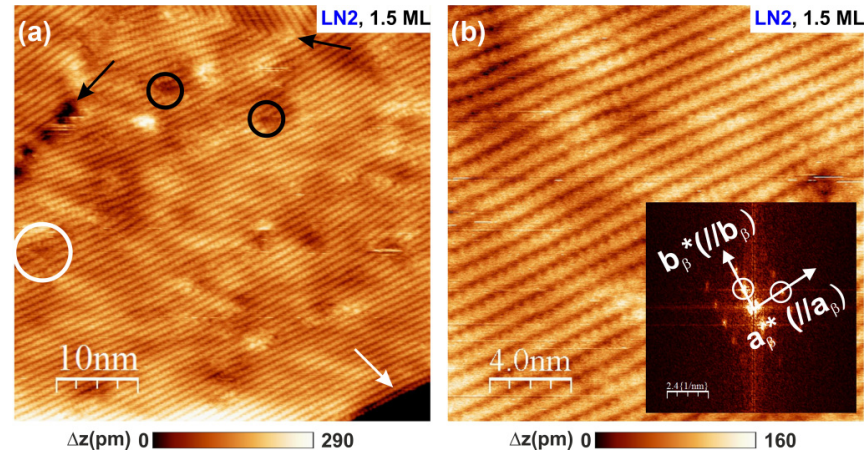

FIG. 7. [(a) and (b)] nc-AFM topographical images of $1.5 \mathrm{ML}$ bis-pyrene on $\mathrm{KCl}(001)$ at $\mathrm{LN} 2$ temperature (a) $50 \times 50$ and (b) $20 \times$ $20 \mathrm{~nm}^{2}$. The molecular u.c. of the $\beta$ phase is well-resolved in (b), both in real and reciprocal spaces (FFT, inset).

$200 \mathrm{~nm}^{2}$ in size). They grow on top of the $\alpha$ phase which behaves as a wetting layer for them. Two types of contrasts are visible on the domains. These are better visible in Fig. 6(b) indicated by brown and green arrows. Figure 6(c) shows the details of the cross-sections A and B shown in Fig. 6(b). Section A shows that the apparent height of some domains reaches (2.20 \pm 0.20$) \mathrm{nm}$ w.r.t. the $\alpha$ phase. This type of domain is referred to as the $\beta$ phase and indicated with brown arrows in Fig. 6(b). Section B shows the $\alpha$ phase (780 pm high w.r.t. the substrate), an $(1.80 \pm 0.20) \mathrm{nm}$ high intermediate domain and a $\beta$-phase domain $[(2.10 \pm 0.20) \mathrm{nm}$ high]. The intermediate domain with a smaller height is referred to as the $\gamma$-phase domain. These domains are indicated with green arrows in Fig. 6(b). Both, $\beta$ and $\gamma$ phases are consistent with a vertical adsorption of the molecules, i.e., with their long axis perpendicularly aligned with respect to the substrate plane. This assumption will be further justified hereafter. It is inferred that the different heights between domains $(\simeq 0.3 \mathrm{~nm})$ stems from the tilt angle the molecules adopt upon adsorption on top of the $\alpha$ phase layer. It is difficult to quantify precisely the percentage of each phase, which is present on the surface. To render our analysis simpler, the mix between $\beta$ - and $\gamma$-phase domains will be approximated to a single mean pseudophase, which will remain referred to as the $\beta$ phase.

To confirm those measurements, additional experiments were performed at LN2 temperature. The morphology of the domains on a large scale is reported in Fig. 6(d). It is essentially similar to the one observed at RT with $\beta$-like domains whose shape remains irregular. The cross section A reported in Fig. 6(e) is consistent with our former assumption. The $\alpha$ phase wetting layer $(\simeq 520 \mathrm{pm}$ high at $\mathrm{LN} 2$ temperature $)$ first develops with molecules adsorbed in flat-lying configuration, then the second layer grows, but with vertically aligned molecules, whose various tilt angles w.r.t. the wetting layer yield essentially two types of domains with two different apparent heights.

In Fig. 7 , the molecular contrast on top of a $\beta$-phase domain is reported (LN2 temperature). The molecular contrast is achieved, however, with a heterogenous mean contrast, which trends to show that the internal structure of the molecular crystal is complex. Nevertheless, the cohesion of the domain must be rather large as can be seen with its sharp edge [cf. 
white arrow in Fig. 7(a)]. In this image, several domains with grain boundaries are visible (cf. black arrows). Their orientation does not seem to be well-defined. Structural defects (black circles) and dislocations (white circle) are visible too. In Fig. 7(b), another high-resolution image is reported. The corresponding FFT (Fig. 7, inset) indicates a rectangular u.c. with dimensions $\left\|\mathbf{a}_{\beta}\right\|=(0.55 \pm 0.05) \mathrm{nm}$ and $\left\|\mathbf{b}_{\beta}\right\|=$ $(0.87 \pm 0.08) \mathrm{nm}$. These dimensions are inconsistent with molecules in a flat lying configuration. Conversely, in a vertical configuration, the molecules can get tightly stacked by $\pi$ stacking. Assuming one molecule per u.c., the packing density for the $\beta$ phase is $\simeq 2.1 \mathrm{~mol} \mathrm{~nm}^{-2}$. This is nearly 10 times larger than the density of the $\alpha$ phase.

This confirms our initial assumption and explains how the growth takes place. After completion of the first ML wherein the molecules are adsorbed in a flat-lying configuration $(\alpha$ phase), the second layer of molecules grows vertically, yielding $\beta$-phase-like domains, which are $\simeq 2 \mathrm{~nm}$ high. These domains have an in-plane crystalline structure. They consist of tightly packed molecules with a rectangular unit cell, likely owing to $\pi$ stacking of their pyrene groups. It is unclear why such a type of growth occurs. In particular, the epitaxy between the $\alpha$ phase and the $\beta$ phase, if any, was not elucidated. Nevertheless, the strong discrepancy between the two types of layers indicates that the $\alpha$ phase results from the interaction, although weak, with the substrate, whereas the $\beta$ phase, whose interaction with the substrate in screened due to the presence of the wetting layer, is more representative of an intrinsic structure of the bulk molecular crystal.

\section{Multilayer regime: $5 \mathrm{ML}$}

Finally, we investigated the multilayer regime. In Fig. 8, the morphology of the bis-pyrene film for a 5-ML coverage [Figs. 8(a) and 8(b)] is presented. On a large scale, the domains keep an irregular shape, visible on the topmost layers. The underlying layers seem nearly complete [cf. Fig. 8(a)]. When zooming, two types of contrasts remain visible on the top part of the domains (data not shown). This observation, combined with the statistical analysis of the height contrast [cf. Fig. 8(b)] indicates that the multilayer regime consists of $\beta$ phases, which are stacked on top of each other and forming nearly complete layers. This might indicate that the growth mode of bis-pyrene on $\mathrm{KCl}$ has a nearly layer-by-layer (Franck van der Merwe) character, partially hindered by a mild Ehrlich Schwoebel barrier [76].

\section{C. nc-AFM imaging of bis-pyrene on $\mathrm{NaCl}(001)$}

The growth of bis-pyrene molecules on $\mathrm{NaCl}(001)$ was investigated with a similar methodology. In the 0.5-1.5-ML regime and at room temperature, the bis-pyrene molecules do not form a stable wetting layer on $\mathrm{NaCl}(001)$. On $\mathrm{KCl}$, the $\alpha$ phase was formed in the lapse of time required to perform the evaporation and transfer the sample into the AFM, i.e., typically 15 minutes and was found to be stable over several days. After similar evaporation conditions on $\mathrm{NaCl}(001)$, the molecules still exhibit a large diffusion several hours after deposition, making imaging difficult. This is illustrated in Fig. 9, which depicts a set of four consecutive images acquired at room temperature in the ML regime over the same area. A

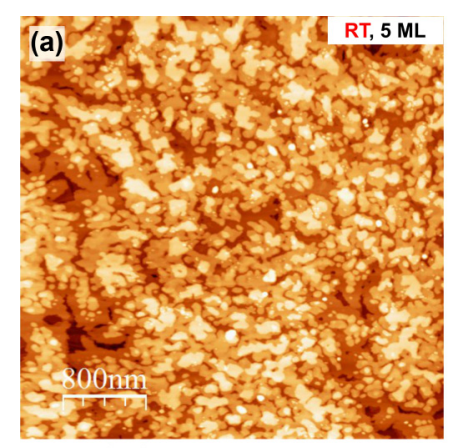

$\Delta z ( \mathrm { nm } ) 0 \longdiv { \square } 1 0$
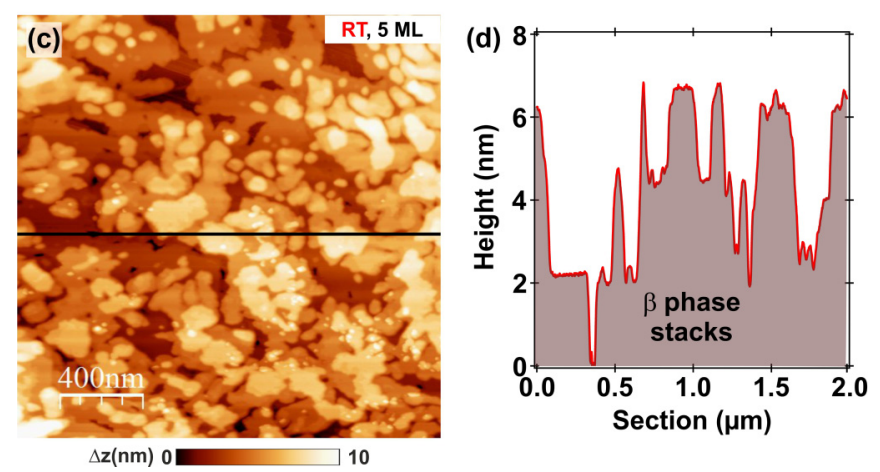

FIG. 8. (a) nc-AFM topographical images of 5 ML bis-pyrene on $\mathrm{KCl}(001)$ at $\mathrm{RT}\left(4 \times 4 \mu \mathrm{m}^{2}\right)$. The molecules form incomplete layers consisting of $\simeq 200 \times 200 \mathrm{~nm}^{2}$ domains. (b) Height distribution of image (a) showing that these are $\beta$-phase-typed domains, which are stacked on top of each other. [(c) and (d)] Other area showing the morphology of the $\beta$-phase domains $\left(2 \times 2 \mu \mathrm{m}^{2}\right)$ and corresponding cross section.

single image lasts $\simeq 20 \mathrm{~min}$. Thus the set depicts an acquisition duration of one hour. The black arrow in the bottom left corner points toward a molecular domain of different type (see discussion hereafter) whose shape remains unchanged. The evolution of the contrast in the surrounding area shows that the molecules diffuse over the surface (cf. circle in Fig. 9). There is a continuous condensation/dissociation of the molecular layer without forming a stable phase.

On other areas, bis-pyrene molecules dewet to form large crystallites [up to $\simeq 15 \mathrm{~nm}$ high, cf. Figs. 10 (a) and 10(b)], socalled aggregates. It is also often observed step edge decoration and medium-sized molecular domains $\left(<200 \times 200 \mathrm{~nm}^{2}\right)$ with a $2.5 \mathrm{~nm}$ apparent height [cf. Figs. 10(c) and 10(d)]. Owing to the results on $\mathrm{KCl}$, those domains are assigned to $\beta$-phase ones, wherein the molecules are tightly packed and stand vertically w.r.t. the $\mathrm{NaCl}$ substrate. The above observations prove that kinetic effects are at play on $\mathrm{NaCl}(001)$ whose consequences influence more the molecular growth than on $\mathrm{KCl}(001)$. However, owing to the relative imaging complexity on that system, these aspects were not investigated further on.

In the 5-ML regime (cf. Fig. 11), as seen on $\mathrm{KCl}$, the molecules form incomplete domains, which stack on top of each other resulting in structures of up to $\simeq 12 \mathrm{~nm}$ high. Here again, the apparent height of these reaches $\simeq 2.5 \mathrm{~nm}$. The fact that the bis-pyrene molecule early trends to form $\beta$-phase domains in the sub-ML regime let us think that the 5-ML 

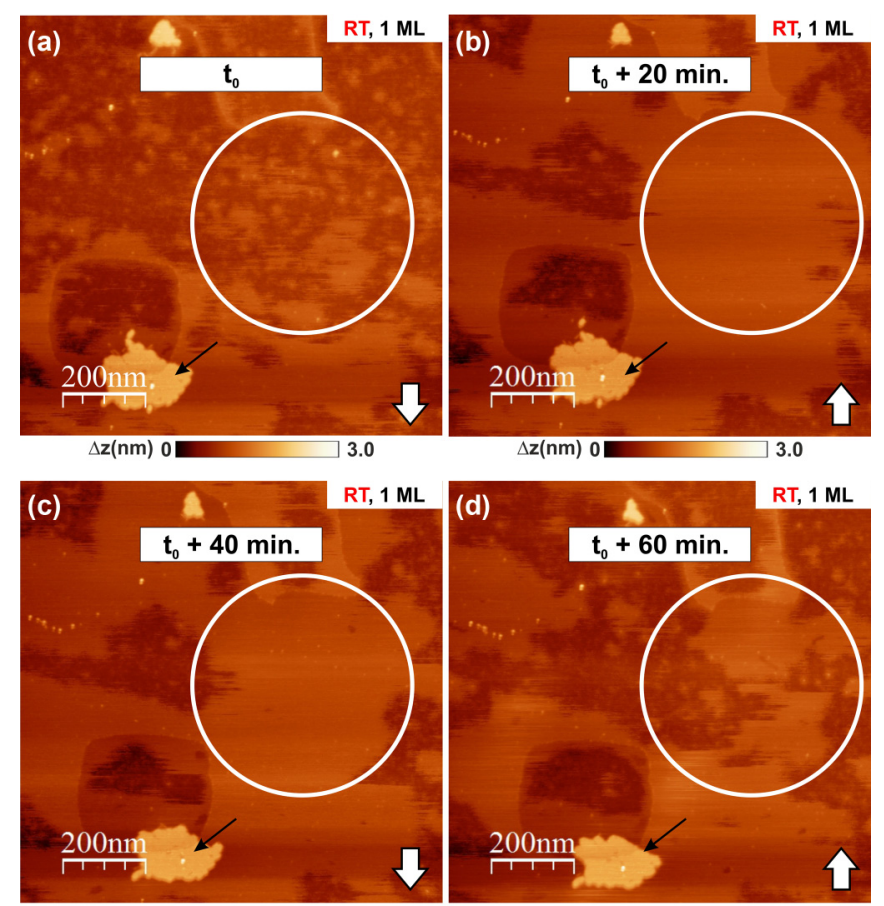

$\Delta z(n m) 0 \square 3.0$

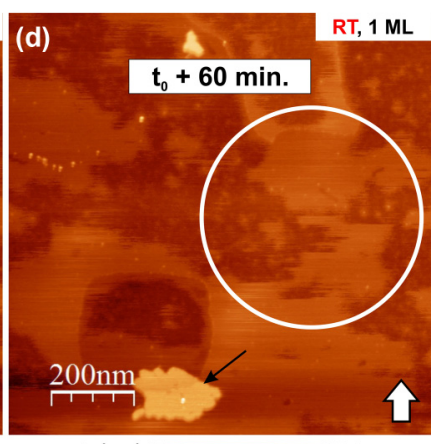

$\Delta \mathrm{z}(\mathrm{nm}) \mathbf{0} \square 3.0$

FIG. 9. nc-AFM topographical image of 1 ML bis-pyrene on $\mathrm{NaCl}(001)$ at RT $\left(1 \times 1 \mu \mathrm{m}^{2}\right)$. Images (a), (b), (c), and (d) have been acquired sequentially over a 20 min duration each.

regime consists of $\beta$-phase-like domains, which are stacked on top of each other.

Thus the resulting morphology of the 5-ML NaCl molecular solid phase is structurally close to the 5-ML $\mathrm{KCl}$ one. Besides, the comparison between the sub-ML and the multilayer regime indicates that on $\mathrm{NaCl}$, the bis-pyrene molecules rather have a Volmer-Weber type of growth.

\section{DRS on $\mathrm{KCl}(001)$}

\section{Experimental spectra}

The DR spectra of the molecules for coverages ranging from 0 to $\simeq 5 \mathrm{ML}$ on $\mathrm{KCl}(001)$, as-calibrated by nc-AFM imaging, are reported in Fig. 12(a). The molecules absorb in a wide band spreading from $380 \mathrm{~nm}$ (limit of detection) up to $550 \mathrm{~nm}$. The fine structure of the band is well-resolved from the ML regime (1 ML, dark blue markers) up to the multilayer regime (5 ML, cyan markers).

In the very early stages of growth, the spectra are broad and featureless. Up to $1 \mathrm{ML}(\alpha$ phase), three main transitions are distinguishable (labelled "1", "2," and "3"), whose characteristics (locations and DRS values) are gathered in Table II. The corresponding peaks are located at $\lambda_{1}=(488 \pm$ 2) $\mathrm{nm}\left[E_{1}=(2.544 \pm 0.010) \mathrm{eV}\right], \lambda_{2}=(459 \pm 2) \mathrm{nm}\left[E_{2}=\right.$ $(2.704 \pm 0.012) \mathrm{eV}]$, and $\lambda_{3}=(432 \pm 2) \mathrm{nm}\left[E_{3}=(2.873 \pm\right.$ $0.013) \mathrm{eV}$. The peak labelled " 2 " is the most prominent, with a DRS value of $(2.82 \pm 0.01) \times 10^{-2}$.

In order to identify at best the various peaks seen in Fig. 12(a), absorption and fluorescence spectra in DCM solvent (cf. Fig. 2) are overlaid with the 1-ML DR spectrum in Fig. 13. The location of the band edge is consistent with the one of the $S_{00} \rightarrow S_{10}$ transition seen in the absorption
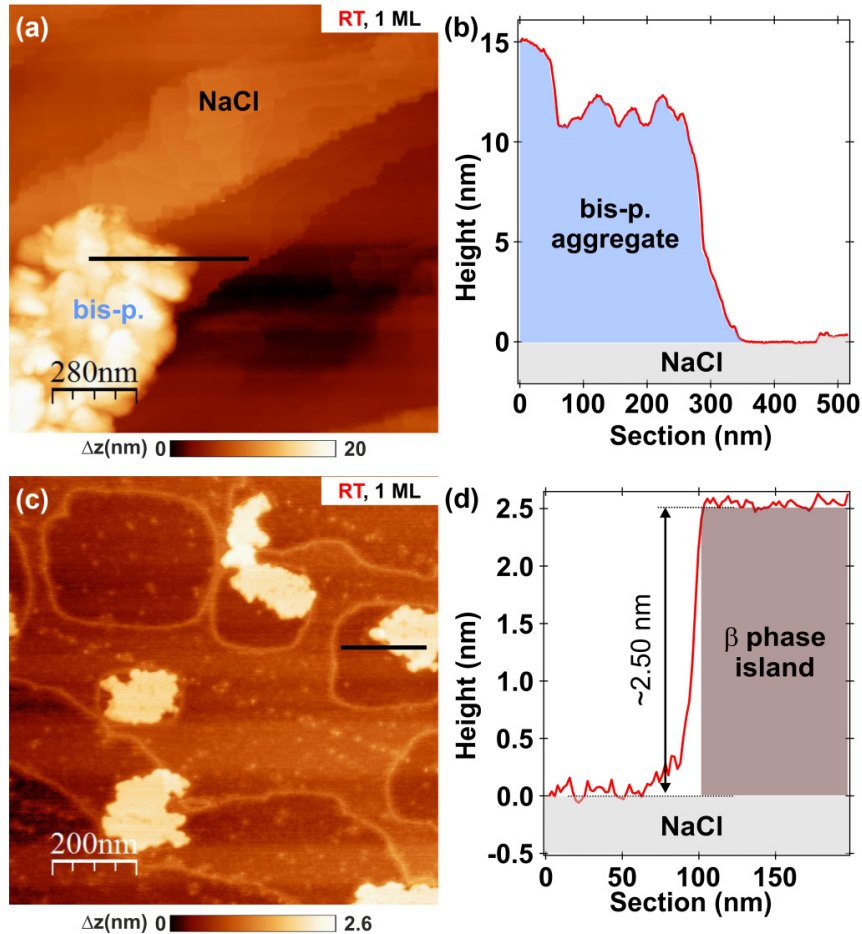

FIG. 10. (a) nc-AFM topographical image of 1 ML bis-pyrene on $\mathrm{NaCl}(001)$ at RT. Locally, the molecules may form large crystallites, so-called aggregates $\left(1.4 \times 1.4 \mu \mathrm{m}^{2}\right)$. (b) Cross-section along the black line in (a). (c) nc-AFM topographical image of 1 ML bis-pyrene on $\mathrm{NaCl}(001)$ at $\mathrm{RT}\left(1 \times 1 \mu \mathrm{m}^{2}\right)$. (d) Cross-section along the black line in (c).

spectra of the molecules in solvent. Thus the corresponding peak labelled "1" in Fig. 12(a) is attributed to the $S_{00} \rightarrow S_{10}$ transition. The position of that transition well matches the corresponding transition of the molecule in solvent, as defined formerly from the overlap between absorption and fluorescence spectra. This observation validates that, upon adsorption on the $\mathrm{KCl}$ substrate, the molecules do not undergo severe structural changes compared to the situation in solvent. Hence the adsorption energy of the molecules on $\mathrm{KCl}$ in the $\alpha$ phase must be weak. The two subsequent
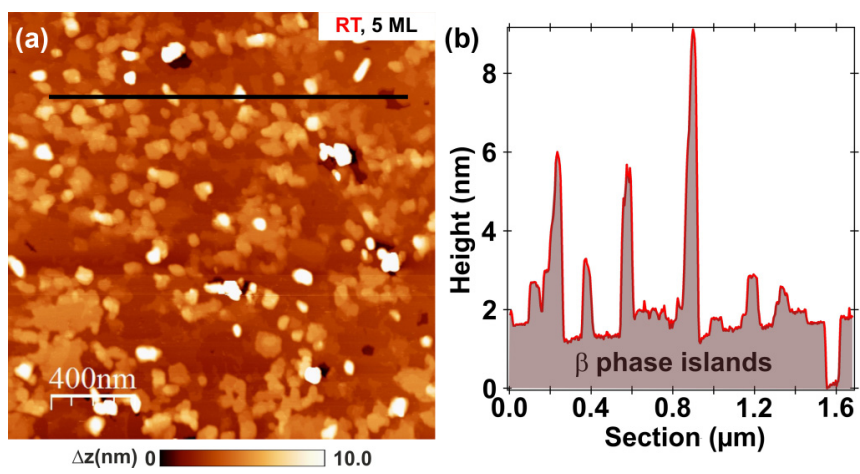

FIG. 11. (a) nc-AFM topographical image of 5 ML bis-pyrene on $\mathrm{NaCl}(001)$ at $\mathrm{RT}\left(2 \times 2 \mu \mathrm{m}^{2}\right)$. The molecules form discontinuous domains, whose morphologies look similar to those seen on $\mathrm{KCl}(001)$ for similar growth conditions. (b) Height profile corresponding to the cross-section visible in (a) (black line). 


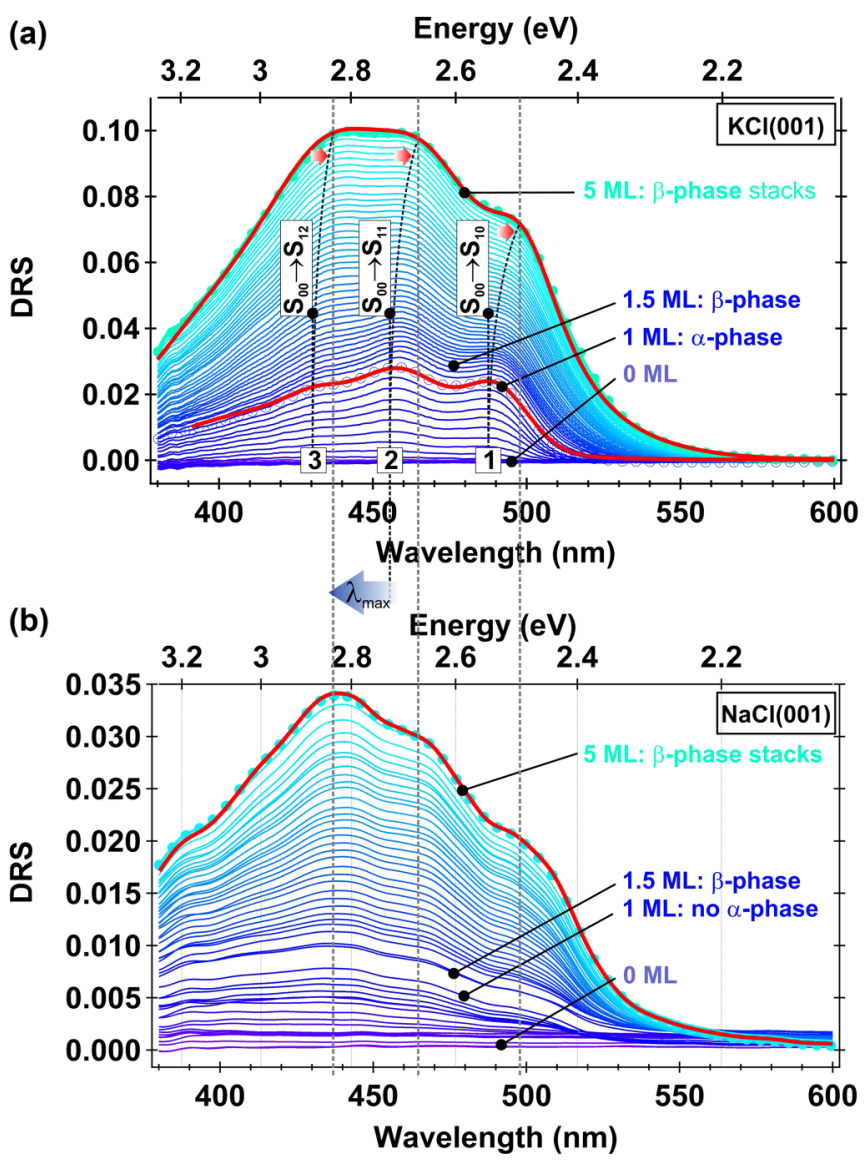

FIG. 12. DR spectra of bis-pyrene on $\mathrm{KCl}(001)$ from 0 to $5 \mathrm{ML}$ (a) and on $\mathrm{NaCl}(001)$ from 0 to $5 \mathrm{ML}$ (b). On $\mathrm{KCl}(001)$, note the blue shift of the $\lambda_{\max }$ depicted by the thick arrow [No. $2(1 \mathrm{ML}) \rightarrow$ No. 3 $(5 \mathrm{ML})]$. The thick red curves depict the fits.

transitions, "2" and "3," are energetically equidistant from each other: $\Delta E_{21}^{1 \mathrm{ML}}=E_{2}^{1 \mathrm{ML}}-E_{1}^{1 \mathrm{ML}}=(0.160 \pm 0.022)$ $\mathrm{eV}, \quad \Delta E_{32}^{1 \mathrm{ML}}=E_{3}^{1 \mathrm{ML}}-E_{2}^{1 \mathrm{ML}}=(0.169 \pm 0.025) \quad \mathrm{eV} \quad(\mathrm{cf}$. Table II). This gap is a bit larger, but comparable to the gap between vibronic replicas, as seen from the fluorescence data in solvents (cf. Fig. 2 and Table I). We therefore attribute them

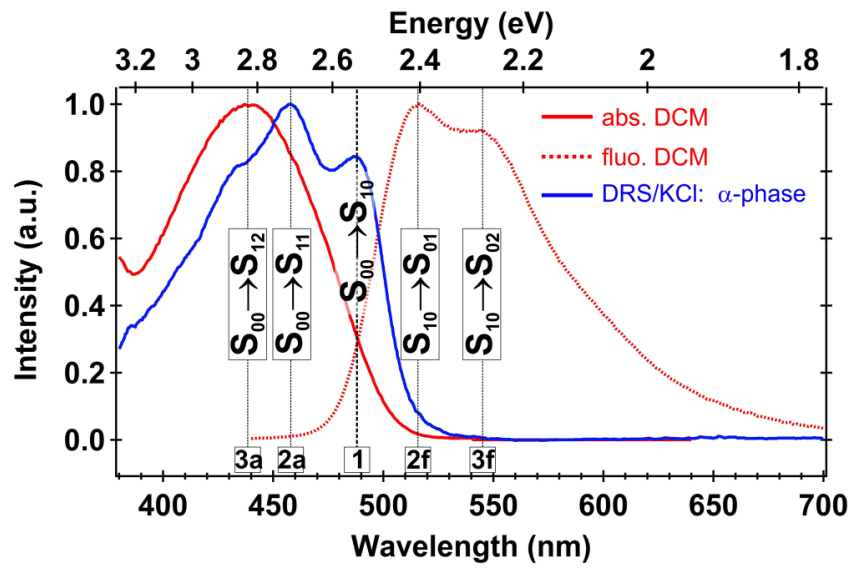

FIG. 13. Comparison between absorption and fluorescence spectra of bis-pyrene in DCM and the 1-ML DR spectrum on $\mathrm{KCl}(001)$. The spectra have all been normalized to their maximum intensity.
TABLE II. Table of the main transitions in the DR spectra of bis-pyrene on $\mathrm{KCl}(001)$ for 1 and $5 \mathrm{ML}$. For transitions 1, 2, and 3, the energy shifts $\Delta E_{1 \mathrm{ML}-5 \mathrm{ML}}$ from 1 to $5 \mathrm{ML}$ are $(-45 \pm 20),(-43 \pm 24)$ and $(-46 \pm 26)$, meV respectively.

\begin{tabular}{|c|c|c|c|c|}
\hline Label & $\begin{array}{l}\text { Transition } \\
\left(S_{00} \rightarrow S_{1 x}\right)\end{array}$ & Regime & $\begin{array}{c}\lambda(\mathrm{nm}) \\
\text { Energy }(\mathrm{eV})\end{array}$ & $\begin{array}{l}\text { DRS value } \\
\left(\times 10^{-2}\right)\end{array}$ \\
\hline \multirow[t]{2}{*}{1} & \multirow[t]{2}{*}{$S_{10}$} & $1 \mathrm{ML}:$ & $\begin{array}{c}(488 \pm 2) \\
(2.544 \pm 0.010)\end{array}$ & $(2.38 \pm 0.01)$ \\
\hline & & $5 \mathrm{ML}:$ & $\begin{array}{c}(496 \pm 2) \\
(2.499 \pm 0.010)\end{array}$ & $(7.29 \pm 0.02)$ \\
\hline \multirow[t]{2}{*}{2} & \multirow[t]{2}{*}{$S_{11}$} & $1 \mathrm{ML}:$ & $\begin{array}{c}(459 \pm 2) \\
(2.704 \pm 0.012)\end{array}$ & $(2.82 \pm 0.01)$ \\
\hline & & $5 \mathrm{ML}:$ & $\begin{array}{c}(466 \pm 2) \\
(2.661 \pm 0.012)\end{array}$ & $(9.91 \pm 0.02)$ \\
\hline \multirow[t]{2}{*}{3} & \multirow[t]{2}{*}{$S_{12}$} & $1 \mathrm{ML}:$ & $\begin{array}{c}(432 \pm 2) \\
(2.873 \pm 0.013)\end{array}$ & $(2.28 \pm 0.01)$ \\
\hline & & $5 \mathrm{ML}:$ & $\begin{array}{c}(439 \pm 2) \\
(2.827 \pm 0.013)\end{array}$ & $(9.98 \pm 0.02)$ \\
\hline
\end{tabular}

to two vibronic replicas of the molecules in the ML, namely, transitions $S_{00} \rightarrow S_{11}$ and $S_{00} \rightarrow S_{12}$. It is remembered that those replicas were not visible in the absorption spectra of the molecule in solvent. This observation is consistent with the assumption that the adsorption of the molecules on the $\mathrm{KCl}$ substrate hinders some of their degrees of freedom, notably libration modes, which makes vibrational features then visible.

Up to $5 \mathrm{ML}$, the peak labelled "3" ( $S_{00} \rightarrow S_{12}$ transition) becomes prominent w.r.t. the two other transitions [value: $(9.98 \pm 0.02) \times 10^{-2}$, cf. Table II]. Compared to the $1-\mathrm{ML}$ case, a moderate redshift of all the transitions (rigid shift) is also to be noticed [ $\simeq-45 \mathrm{meV}$, cf. Table II and black-dotted lines in Fig. 12(a)]. Nonetheless, the overall shape of the absorption band changes and a blueshift is observed, which may qualitatively be seen by the shift of the $\lambda_{\max }$ of the spectra. This trend is similar to the one seen in ACN compared to other solvents and might trace the first aggregation stages of the condensed molecular phase. For that coverage, one may also notice that the band edge above $500 \mathrm{~nm}$ (the tail of the DR spectrum) decays more slowly than the corresponding band edge at $1 \mathrm{ML}$. The rapid decay of the absorption band edge of the 1-ML DR spectrum will be referred to as a relative "sharpness" of the spectrum.

\section{Fitting the DR spectra}

Owing to the nonlinear nature of Fresnel's equations, a DR spectrum is not expected to simply scale linearly with the thickness of the adlayer. Peak shifts and/or modification of their relative intensities can also be envisaged as the adlayer thickness increases. Therefore attention is to be paid on how to extract intrinsic parameters, characterizing only the absorbing entities of the layers, from a DRS measurement. In the case of the transition from $\alpha$ to $\beta$ phase, the targeted intrinsic quantities, irrespectively from the DRS formalism, are the oscillator strength and the exact peak position of each vibronic replica.

This is all the more true with anisotropic media. Now, from an optical point of view, the bis-pyrene molecule is strongly anisotropic as its transition dipole moment is aligned 
with the long molecular axis [77]. Therefore the absorption of the molecular layers $(\alpha$ or $\beta$ ) must be treated with an anisotropic formalism of their dielectric function. The most generic approach to optical properties is given by the case of biaxial crystals. However, this case is hardly tractable, as seen for instance with ellipsometry formalism $[78,79]$. Here, the nc-AFM structural results incite us to use a simplified framework. Owing to the substrate symmetry $\left(A_{4}\right.$ axis along the $\langle 001\rangle$ direction, i.e., the $z$ axis), four epitaxy variants of the molecular layers are expected, with $25 \%$ equiprobability each. Therefore the adsorbed molecular crystals have in average, a quadratic symmetry. Thus we simplify the problem by assuming that the bis-pyrene molecular layers optically behave as uniaxial crystals, with their optic axis (i.e., growth direction) perpendicular to the (001) substrate plane [80,81]. A thorough fitting procedure of the recorded DR spectra in the uniaxial approximation was developed, which is detailed in the Appendix.

The nc-AFM structural data have established that bispyrene molecules grow in two steps, with first the development of the $\alpha$ phase (1-ML $\alpha$ case) and then a quasi-layer-by-layer growth mode of $\beta$-phase domains (5-ML $\beta / \alpha$ case). In the $\alpha$ phase, the molecules are lying flat, which means that their transition dipole moment is perpendicular to the optic axis. Thus, in a set of axes given by the substrate directions: $x=$ $\langle 100\rangle, y=\langle 010\rangle$, and $z=\langle 001\rangle$, the dielectric tensor for the $\alpha$-phase uniaxial crystal has the form

$$
\overline{\bar{\epsilon}}_{\alpha}=\left(\begin{array}{ccc}
\widetilde{\epsilon}_{\alpha, \bmod }^{\perp}(\lambda) & 0 & 0 \\
0 & \widetilde{\epsilon}_{\alpha, \bmod }^{\perp}(\lambda) & 0 \\
0 & 0 & \epsilon_{\alpha}^{\|, \infty}
\end{array}\right),
$$

where the indices $\perp$ and $\|$ depict the perpendicular and parallel directions to the optic axis. $\widetilde{\epsilon}_{\alpha \text {,mod }}^{\perp}(\lambda)$ stands for the $\perp$ component of the dielectric tensor. It is a complex quantity to account for the absorption of the layer in the $(x, y)$ plane and its formal expression is given by the retained fit model (cf. below). Because the molecules are not expected to absorb light in the $z$ direction, $\epsilon_{\alpha}^{\|, \infty}$ is a real constant.

Conversely, the dielectric tensor for the $\beta$-phase uniaxial crystal must have the form

$$
\overline{\bar{\epsilon}}_{\beta}=\left(\begin{array}{ccc}
\epsilon_{\beta}^{\perp, \infty} & 0 & 0 \\
0 & \epsilon_{\beta}^{\perp, \infty} & 0 \\
0 & 0 & \widetilde{\epsilon}_{\beta, \text { mod }}^{\|}(\lambda)
\end{array}\right),
$$

where $\widetilde{\epsilon}_{\beta, \bmod }^{\|}(\lambda)$ stands for the $\|$ component of the dielectric tensor. It is a complex quantity because, in $\beta$-phase configuration, the molecules absorb the light in that direction only. Its formal expression has the form given by the retained fit model here as well. In the $(x, y)$ plane, $\epsilon_{\beta}^{\perp, \infty}$ is a real constant.

The most common approach to the dielectric function for a homogeneous medium is to mimic each absorption peak by a Lorentz oscillator (Lorentz oscillator model, LOM). Considering three transitions here (i.e., the vibronic progression), the LOM for the dielectric function of the molecular adlayer has the form

$$
\tilde{\epsilon}_{\mathrm{LOM}}(\lambda)=\epsilon^{\infty}+\sum_{j=1}^{3} \frac{f_{j}}{1-\left(\frac{\lambda_{j}}{\lambda}\right)^{2}+i \gamma_{j}\left(\frac{\lambda_{j}}{\lambda}\right)},
$$

with $\epsilon^{\infty}$ a corrective term accounting for the dielectric behavior in the deep UV. This function allows for extracting the wavelength $\lambda_{j}$ and the dimensionless parameter $\gamma_{j}$ concealing the electronic state lifetime and the oscillator strength $f_{j}$ of each transition.

However, it is impossible to fit the DR spectrum of the 1-ML $\alpha$ case by using the LOM as the input model for $\widetilde{\epsilon}_{\alpha, \text { mod }}^{\perp}(\lambda)$. In particular, the fit fails in reproducing the sharpness of the absorption band edge of the DR spectrum around $500 \mathrm{~nm}$. To achieve good fitting quality, a set of Lorentz oscillators per transition has to be considered instead of a single oscillator. We arbitrary chose a distribution of oscillators whose absorption wavelengthes spread around each absorption transition of the vibronic progression and whose strengthes follow a Gaussian envelope, namely,

$$
\begin{aligned}
\tilde{\epsilon}_{\alpha, \text { inh }}^{\perp}(\lambda)= & \epsilon^{\infty}+\frac{1}{\sqrt{2 \pi} \sigma} \sum_{j=1}^{3} \int_{-\infty}^{+\infty} e^{-\lambda_{s}^{2} / 2 \sigma^{2}} \\
& \times \frac{f_{j}}{1-\left(\frac{\lambda_{j}-\lambda_{s}}{\lambda}\right)^{2}+i \gamma_{j}\left(\frac{\lambda_{j}-\lambda_{s}}{\lambda}\right)} d \lambda_{s} .
\end{aligned}
$$

The fit is performed by using the dielectric tensor given in Eq. (3), but with the expression given by Eq. (6) for the $\perp$ component of the dielectric function of the $\alpha$ phase instead of Eq. (5) (cf. Appendix for details). The broadening of the transitions resembles the inhomogeneous broadening reported for dye molecules in solvents [82]. Therefore this model is referred to as the "inhomogeneous model." This means that the first layer of molecules in contact with the $\mathrm{KCl}$ surface are sensitive to several interactions (moleculemolecule and molecule-surface), and/or conformations due to thermal agitation, which, in average, do not change the location of their transitions between electronic levels, but nevertheless broadens their natural width. This was evidenced by the structural imaging (cf. Sec. III B 1).

For the fit of the 5-ML $\beta / \alpha$ DR spectrum, a three interfacessystem consisting of two semi-infinite isotropic media (vacuum and substrate) intercalated with two uniaxial crystals (cf. Fig. 17 in the Appendix) is considered. The optical response of the as-defined global composite system is described by means of the transfer matrix method (TMM) $[83,84]$ (cf. Appendix for details). The optical response of the $\beta$-phase layer was described by the dielectric tensor of Eq. (4) with the LOM given by Eq. (5) for the $\|$ component of the dielectric function. The optical response of the $\alpha$-phase layer remains described by the one derived from the fit of the 1-ML $\alpha$ case.

Both models (LOM, inhomogeneous model), treated in an anisotropic approach of the adlayers and combined with the TMM formalism, not only allow us to locate unambiguously the positions of the peaks, but to extract the complete dielectric function of the adlayers and their equivalent optical thicknesses as well. The fits corresponding to both 1-ML $\alpha$ and 5-ML $\beta / \alpha$ cases have been overlaid in Fig. 12(a) (thick red curves). They match the experimental spectra with a good accuracy. The corresponding parameters are reported in Table III. In the 1and 5-ML regimes, the values of the fitted thicknesses are $d_{\alpha}=$ $(1.1 \pm 0.2) \mathrm{nm}$ and $d_{\beta / \alpha}=(10.2 \pm 0.5) \mathrm{nm}$, respectively. These are in good agreement with the corresponding apparent heights of the molecular structures determined by nc-AFM.

The fits confirm the experimental trends. (1) The oscillator strength of the $S_{00} \rightarrow S_{12}$ transition becomes prominent in the 
TABLE III. Table of the fitted parameters of the DR spectra for the 1-ML $\alpha$ and 5-ML $\beta / \alpha$ cases on $\mathrm{KCl}(001)$ and for 5-ML $\beta$ islands on $\mathrm{NaCl}(001)$. For $\mathrm{KCl}(001)$, in the $1-\mathrm{ML} \alpha$ case, the anisotropic TMM formalism using an inhomogeneous model for the $\perp$ component of the dielectric function was used (cf. text). The fitted layer thickness is $(1.1 \pm 0.2) \mathrm{nm}$. In the 5-ML $\beta / \alpha$ case, the anisotropic TMM formalism using the LOM for the $\|$ component of the dielectric function was used, with a fitted thickness = $(10.2 \pm 0.6) \mathrm{nm}$. The refractive index was $n_{\mathrm{KCl}}=1.48$. For $\mathrm{NaCl}(001)$, the anisotropic TMM formalism using the LOM for the $\|$ component of the dielectric function in the EMT approximation was used. The fitted layer thickness is $(7.1 \pm 0.6) \mathrm{nm}$. The refractive index was $n_{\mathrm{NaCl}}=1.53$. For both substrates, the incidence light angle was $\theta_{0}=65^{\circ}$, consistently with the setup.

\begin{tabular}{lccccc}
\hline \hline $\begin{array}{l}\text { Regime (fit model) } \\
\epsilon^{\perp, \infty}, \epsilon^{\|, \infty}\end{array}$ & Label & $\begin{array}{c}\text { Transition } \\
\left(S_{00} \rightarrow S_{x y}\right)\end{array}$ & $\begin{array}{c}\lambda_{j} \\
(\mathrm{~nm})\end{array}$ & $\begin{array}{c}f_{j} \\
()\end{array}$ & $\begin{array}{c}\gamma_{j} \\
()\end{array}$ \\
\hline \multicolumn{5}{c}{$\mathrm{KCl}(001)$} \\
$1 \mathrm{ML} \alpha$ (inhomo.) & 1 & $S_{10}$ & 490.1 & 0.0024 & 0.019 \\
$\epsilon_{\alpha}^{\perp, \infty}=(1.30 \pm 0.01)$ & 2 & $S_{11}$ & 459.8 & 0.0062 & 0.026 \\
$\epsilon_{\alpha}^{\|, \infty}=(1.25 \pm 0.01)$ & 3 & $S_{12}$ & 431.9 & 0.0017 & 0.024 \\
$5 \mathrm{ML} \beta / \alpha(\mathrm{LOM})$ & 1 & $S_{10}$ & 515.9 & 0.0698 & 0.073 \\
$\epsilon_{\beta}^{\perp, \infty}=(1.60 \pm 0.01)$ & 2 & $S_{11}$ & 480.4 & 0.0149 & 0.062 \\
$\epsilon_{\beta}^{\|, \infty}=(1.10 \pm 0.01)$ & 3 & $S_{12}$ & 455.8 & 0.0848 & 0.180 \\
& & $\mathrm{NaCl}(001)$ & & & \\
$5 \mathrm{ML} \beta(\mathrm{EMT}-\mathrm{LOM})$ & 1 & $S_{10}$ & 510.8 & 0.0696 & 0.072 \\
$\epsilon_{\beta}^{\perp, \infty}=(1.70 \pm 0.01)$ & 2 & $S_{11}$ & 480.6 & 0.0210 & 0.061 \\
$\epsilon_{\beta}^{\|, \infty}=(1.10 \pm 0.01)$ & 3 & $S_{12}$ & 456.7 & 0.0805 & 0.167 \\
\hline \hline
\end{tabular}

5-ML regime, whereas it was the weakest at 1 ML. (2) They account for the rigid redshift of each transition in the spectra in the 5-ML $\beta / \alpha$ case as well. For this case, we note the important discrepancy between the positions of the experimental peaks and the fitted ones (cf. Tables II and III, respectively), of up to $+20 \mathrm{~nm}$. The origin of such an effect is not clear. Because the positions of the experimental peaks and the fitted ones are quite similar in the 1-ML $\alpha$ case, we might speculate that the DRS signal is mainly sensitive to the $s$-polarized component of the incoming light. Indeed, it is this component which is aligned with the transition dipole moment of the molecules in the $\alpha$ phase. Conversely, in the $\beta$ phase, the transition dipole moment of the molecules rather couples to the $p$ component of the light. (3) At last, the band edge sharpness in the 1-ML regime is well-reproduced by the inhomogeneous model. In Ref. [51], a similar sharpness of the absorption band edge (i.e., at large $\lambda$ ) was reported in DR spectra measured on PTCDA molecules adsorbed on $\mathrm{KCl}(001)$. The authors inferred that the important structural ordering of the layers was responsible for such an effect. When compared to our case, this result points the difficulty to interpret the DR spectra ex abrupto, i.e., without further ideas on the layer structure.

\section{E. DRS on $\mathrm{NaCl}(001)$}

\section{Experimental spectra}

On $\mathrm{NaCl}(001)$, the morphology of the DR spectra from 0 to a $\simeq 5$-ML coverage is significantly different [cf. Fig. 12(b)]. The three transitions that were visible for $1 \mathrm{ML}$ on $\mathrm{KCl}(001)$ are distinguishable, but not well-defined. Each transition is there much broader. This is consistent with the molecular gas phase seen in nc-AFM and its time evolution. The resulting DR spectrum for that coverage is therefore hardly interpretable since it combines both, the optical fingerprint of the gas phase and the ripening of the first $\beta$-phase islands. One notices that it is the $S_{00} \rightarrow S_{12}$ transition whose strength is the more pronounced. Beyond $1 \mathrm{ML}$ and up to $5 \mathrm{ML}$, the three transitions become better resolved, but without any intensity reversal however. Their positions do not evolve neither, but they coincide with those of the 5-ML case on $\mathrm{KCl}(001)$ (grey dotted lines in Fig. 12). Those trends, oppositely with $\mathrm{KCl}(001)$, lead to figure out that the DR spectra can be interpreted as resulting from $\beta$-phase islands only, whatever the coverage rate.

\section{Fitting the DR spectra}

Following the methodology on $\mathrm{KCl}(001)$, we want to fit the 5-ML DR spectrum, which is the most structured one. Because the growth mode was found to be different between $\mathrm{KCl}(001)$ and $\mathrm{NaCl}(001)$ (quasi-van der Merwe and VolmerWeber, respectively), the fit model is adapted. The incomplete molecular film is interpreted as an inhomogeneous effective medium (islands+vacuum) and is treated out of an homogeneization process (i.e., the search for an effective equivalent dielectric function) by using the Maxwell-Garnett effective medium theory (EMT) [85,86]. Here, the EMT approximation is applied independently on both $\perp$ and $\|$ components to keep the anisotropic description of the dielectric tensor [87]. Each effective dielectric function is given by

$$
\epsilon_{\mathrm{EMT}}^{\perp, \|}(\lambda)=\epsilon_{h}(\lambda) \frac{\epsilon_{h}(\lambda)+[v(1-f)+f]\left[\epsilon^{\perp, \|}(\lambda)-\epsilon_{h}(\lambda)\right]}{\epsilon_{h}(\lambda)+v(1-f)\left[\epsilon^{\perp, \|}(\lambda)-\epsilon_{h}(\lambda)\right]},
$$

Here, $\epsilon_{h}(\lambda)=1$ stands for the dielectric function of the host material (the vacuum) and $\epsilon^{\perp, \|}(\lambda)$ stands for the dielectric function of the inclusions of matter organized into islands. For $\epsilon^{\|}(\lambda)$, we chose a LOM with three transitions. In the EMT, the shape of the islands is accounted for by the depolarization factor $v$ in Eq. (7). We chose $v=1 / 3$, which corresponds to spherical islands. The filling parameter $f$, with $0<f<1$, sets the percentage of matter to be considered within the host material. Based on the nc-AFM structural data (cf. Fig. 11), we have estimated that a $65 \%$ rate of matter was quite representative of the 5-ML case, thus $f=0.65$.

The EMT approximation allows the fit to be performed with a good accuracy [thick red curve in Fig. 12(b)]. The as-derived parameters are reported in Table III. The corresponding fitted thickness is $(7.1 \pm 0.6) \mathrm{nm}$, in reasonable agreement with the estimated height of the $\beta$-phase islands determined by nc-AFM.

The fits strengthen our preliminary conclusions here too. Although the difference in shape between DR spectra measured on $\mathrm{KCl}(001)$ and $\mathrm{NaCl}(001)$ is obvious, the fact that fairly similar fit parameters are derived (cf. Table III) testifies that the molecular packing mainly consists of $\beta$-phase domains, but in different growth mode regimes. 


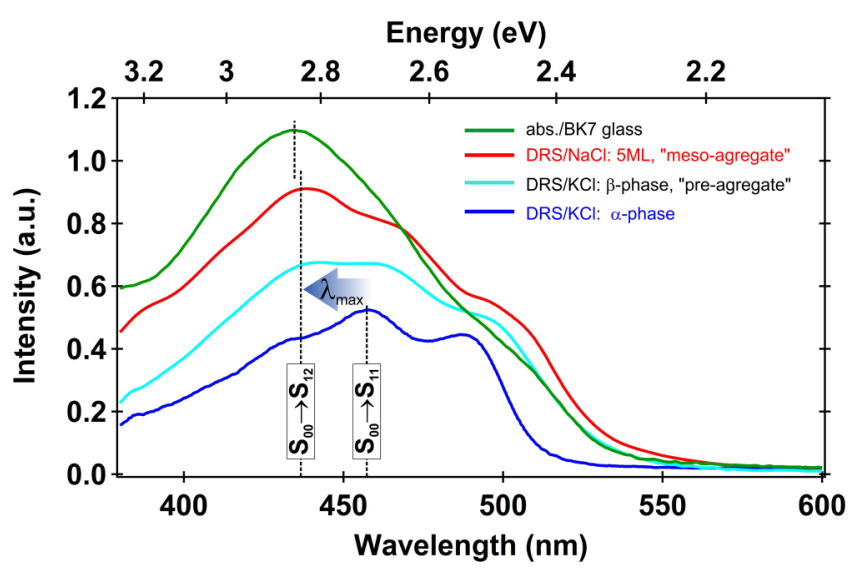

FIG. 14. Illustrative sketch showing the hypsochromic effect with the aggregation of bis-pyrene molecules from its early stages (DR spectrum of the $\alpha$ phase on $\mathrm{KCl}$, blue curve), the preaggregation stages (5-ML DR spectrum of the $\beta$ phase on $\mathrm{KCl}$, cyan curve), mesoaggregates (5-ML DR spectrum of the $\beta$ phase on $\mathrm{NaCl}$, red curve), and further extended aggregates (absorption spectrum on BK7 glass, after Ref. [37], courtesy from the authors). The spectra intensities have been arbitrarily scaled for comparison purpose.

\section{DISCUSSION}

\section{A. On the limit of the fit model}

In order to fit the $\mathrm{DR}$ spectra on $\mathrm{KCl}(001)$ (5-ML $\beta / \alpha$ case), the multilayered global composite system that was used was treated in the TMM formalism assuming flat interfaces. Experimentally, the quasi-layer-by-layer growth mode yields rough interfaces [cf. Figs. 8(a) and 8(c)], whose roughness could be estimated out of the thickness of a single $\beta$-phase layer $(\simeq 2 \mathrm{~nm})$. Such a roughness might influence the fit. We therefore have reconsidered the TMM approach in the EMT approximation to account for rough interfaces (cf. Appendix). It is found that, even a relatively large surface roughness (4 $\mathrm{nm}$ ) neither significantly influences the shape, nor the derived fit parameters.

The relevance of the EMT for tackling optical properties of rough and/or inhomogeneous materials is a delicate issue. Although largely used in various applications despite some recognized limitations [88-92], EMT seems to be the last resort to gain reliable results in a tractable way on disordered composite systems.

\section{B. Signature of the aggregation in the DR spectra}

The absorption spectra (cf. Fig. 1, and the synopsis in Fig. 14) reveal that the optical signature of bis-pyrene, once stacked, is characterized by a hypsochromic effect, as the one reported with $\mathrm{H}$ aggregates. The blueshift of the $\lambda_{\max }$ peak observed between isolated molecules (all solvents except ACN, and $\alpha$ phase) and aggregated ones ( $5 \mathrm{ML}$ on $\mathrm{KCl}$, or islands on $\mathrm{NaCl}$ ) is clearly interpreted as stemming from a strong increase of the $S_{12}$ transition rather than from a rigid blueshift of the entire spectra. In Fig. 14, a sketch is reported to illustrate that, where both, 1-ML KCl, 5-ML KCl, and 5-ML NaCl DR spectra are reported. As early evidenced by Kasha [93,94], the hypsochromic effect results from the coupling between parallel transition dipole moments of, at least, two neighboring excited molecules. This kind of coupling is naturally expected when, for instance, planar (or quasiplanar) molecules are closely stacked into a dimeric-like parallel arrangement (i.e., wherein molecules are oriented in the same way, with their molecular planes being face to face and parallel). Of course, if all the molecules in the crystal are parallel (cf. Ref. [95] for instance) hypsochromism is expected as well, but this situation is too restrictive. In fact, if a sufficiently large amount of dimeric arrangements develop at a short scale in any complex crystalline structure, then the absorption spectrum of the global solid is expected to be blueshifted.

Regarding the case of the $\mathrm{H}$ aggregate precipitated in $\mathrm{ACN}$, we can just conclude that bis-pyrene molecules trend to pack, at least at a short scale, in a parallel configuration resulting from $\pi-\pi$ interactions (cf. Sec. III A 3). This was also evidenced by $\mathrm{x}$-ray diffraction analyzes on bis-pyrene single crystals [38]. Thus it is intuited that the dimericlike parallel arrangement of bis-pyrene molecules acts as a base motif which spatially self-organizes with more or less complexity to form various crystalline structures as, for example, the $\mathrm{SiO}_{4}^{4-}$ tetrahedron does in the various silicate structures.

Bearing in mind this general trend, the optical and structural data of bis-pyrene molecules on $\mathrm{KCl}$ or $\mathrm{NaCl}$ are now discussed in more details. On $\mathrm{KCl}(001)$, the evolution of the thickness and/or of the roughness of the molecular top layers (1-ML $\rightarrow$ 5-ML growth mode) can neither explain the corresponding intensity reversal leading to the $\lambda_{\max }$ blueshift, nor the rigid redshift of each individual vibronic peak. For this, it is mandatory to consider two distinct dielectric functions ( $\alpha$ - and $\beta$-phase-related ones) with distinct sets of oscillator strengths in the LOM. This testifies that upon the phase they are part of, the molecules behave as two distinct absorption centers. We interpret this as resulting from the transition between the absorption of equivalent monomers (molecules in the $\alpha$ phase) to the absorption of dimers or oligomers (molecules in the 5-ML $\beta$ phase). In other words, the $\alpha$ phase, within which the molecules exhibit some mobility due to partial disorder into the layer, has the optical signature of molecules in diluted solution (monomer), however, including vibronic replicas due to flat-lying adsorption. The 5-ML regime (stacks of $\beta$ phases) has the optical signature of a condensed molecular phase which is the trace of preaggregation stages (dimers, then oligomers). The transition from monomer to oligomer is the framework of excimers theory, including $\mathrm{H}$ aggregates and their excitonic interactions [70,71,93-100]. The reconfiguration of the absorption spectra and hence of the molecular levels by dipolar transition moment coupling is expected then. As shown in Sec. III B 2, the packing of the $\beta$ phase can clearly be understood by considering a dimerlike base motif. For pyrenebased molecules, the transition dipole moment is aligned with the long molecular axis [77], thus in the $\beta$ phase, the coupling between the transition dipole moments is necessarily parallel-like and consequently, hypsochromism is expected (H-aggregate behavior). The comparison between DR spectra of the $\alpha$ phase (monomer) and the $\beta$ phase (oligomers) exhibits a net blueshift of the $\lambda_{\max }$, readily understood by an increase of the coupling strength from $\alpha$ to $\beta$ phase due to their respective crystallographic structure.

The 5-ML DR spectra on $\mathrm{NaCl}(001)$ yield similar oscillator strengthes as those derived from the 5-ML $\beta / \alpha \mathrm{KCl}(001)$ 
case, which testifies that the absorption center is $\beta$-phase-like. In Fig. 14, the absorption spectrum of a 50-nm bis-pyrene thick-film grown on a BK7 glass substrate was appended (green curve), as reported in Ref. [37] (cf. Fig. 2 of the reference, red curve, courtesy from the authors). This case is interesting because, on an amorphous material such as glass, no epitaxy is expected. Thus the grown-continuous film has certainly a polycrystalline structure resulting from the coalescence of $\beta$-phase islands. The adsorption of the molecules on BK7 is therefore expected to be more alike the one on $\mathrm{NaCl}$, which can be viewed as an asymptotic case of our 5-ML $\mathrm{NaCl}$ DR spectrum. As sketched in Fig. 14, the inversion of peaks intensity yielding the blueshift seems to be a general trend with bis-pyrene molecules.

\section{On the rigid redshift of the DR spectra on $\mathrm{KCl}(001)$}

Because the $\beta$ phase yields an excitonic coupling, one might expect the excitonic length to increase with the thickness layer. Such an effect naturally yields a redshift of the absorption position $[69,101]$. However, nc-AFM imaging shows that the molecules get stacked laterally forming large domains $\left(>200 \times 200 \mathrm{~nm}^{2}\right)$, with a quasi-layer-by-layer growth. Thus the exciton is rather expected to diffuse within the layer, i.e., laterally, and not (or a few) between vertical stacks. Therefore it is difficult to assess the influence of the thickness on the redshift.

Another potential microscopic explanation would be to invoke the strengthening of gas to solid shift effects [95] (related to the stabilization of the excited state in condensed phase) with the thickness. From a mesoscopic point of view, and according to the Lorentz approach, the electric field acting on a molecule in an isotropic medium and causing its polarization, does not equal the mean field (i.e., satisfying Maxwell's equations), but rather the local one which self-consistently includes the polarization field. Then, the well-known Lorenz-Lorentz (or Clausius-Mossoti) formula giving the dielectric constant as a function of the polarizabilities of gaseous molecules tells that, in any molecular crystal, a transition is redshifted [69] when compared to the same transition in the gas phase. However, since the DR spectra on 5-ML $\mathrm{KCl}(001)$ and islands on $\mathrm{NaCl}(001)$ are both described with the same dielectric function (the $\beta$-phase one) and that no redshift is observed on $\mathrm{NaCl}(001)$ from 1 to $5 \mathrm{ML}$, i.e., while making the islands bigger, it seems difficult to ascribe a condensed phase effect on $\mathrm{KCl}(001)$ and not on $\mathrm{NaCl}(001)$.

A more intuitive interpretation of the redshift is to consider that the intrinsic $\beta$-phase optical response is the same regardless of its deposited thickness. A similar approach, applied to other organic systems, is reported in Ref. [56]. It is inferred that the measured DR spectrum on $\mathrm{KCl}(001)$ results from a combination of the optical response of both, the $\alpha$ phase and the growing film of $\beta$-phase layers. The contribution of the $\alpha$ phase remains constant (1 ML), whereas the contribution of the $\beta$-phase layers gets more and more pronounced as the thickness of the film increases. This result was simulated by means of the TMM and quantitatively reproduces the experimental trends (cf. Appendix).

\section{CONCLUSION}

We report a set of joint structural and optical analyzes of self-assemblies of $\pi$-conjugated bis-pyrene derivatives upon adsorption on two types of bulk insulators. The structural analysis is performed from the subML regime to $5 \mathrm{ML}$ with molecular resolution by means of nc-AFM. In situ optical spectroscopy is performed by means of differential reflectance spectroscopy. That work takes place in a strong methodological and theoretical background where both, the solvatochromism and the fluorescence of the molecular tectons is first assessed to make the quantitative interpretation of the DR spectra more accurate.

On $\mathrm{KCl}(001)$, in the sub-ML regime and at room temperature, the molecules interact with the substrate due to a weakly corrugated van der Waals background and diffuse on the surface over large distances. Then, they nucleate from step edges and condense into large domains ( $\alpha$ phase), whose supramolecular structure forms an epitaxial supercell with the substrate. The structure can only be unravelled at LN2 temperature, which lowers the molecular mobility whereas at RT, the layer presents structural disorder. The squared u.c. presents a weak packing density of $\simeq 0.2 \mathrm{~mol} \mathrm{~nm}^{-2}$. The apparent height of the domains $[\simeq(780 \pm 40) \mathrm{pm}$ at $\mathrm{RT},(520 \pm 20) \mathrm{pm}$ at LN2] indicates that the molecules are adsorbed with their long axis lying parallel to the substrate plane, however, in a configuration which makes them appearing high. Both, this apparent height and the difficulty to achieve high resolution at $\mathrm{RT}$ is attributed to the motion of the alkyl chains due to thermal agitation, which prevents the tip from developing a stable, local, interaction with each molecule. The $\alpha$ phase grows until full completion. Beyond the ML regime, the structure of the layer changes ( $\beta$ phase). The new structure has a rectangular u.c. wherein the molecules are all parallel [i.e., with their long axis perpendicular to the $\mathrm{KCl}(001)$ plane] and with a packing density of $\simeq 2 \mathrm{~mol} \mathrm{~nm}^{-2}$. The nc-AFM imaging reveals that, even at room temperature, the $\beta$-phase domains are rather well-ordered. From 1 to $5 \mathrm{ML}$, a quasi-layer-by-layer growth mode of $\beta$-typed domains is observed.

On $\mathrm{NaCl}(001)$ in the $\mathrm{ML}$ regime, the molecules exhibit a large diffusion too, however without the possibility to develop a stable supramolecular phase (no $\alpha$ phase). This points towards a weaker interaction with the substrate than on $\mathrm{KCl}(001)$. They either form small $\beta$-phase-like islands, which are stable over durations large enough to image them by nc-AFM, or dewet to form large aggregates. Up to $5 \mathrm{ML}$, a Vomer-Weber growth mode of $\beta$-typed domains is observed.

On $\mathrm{KCl}(001)$ and $\mathrm{NaCl}(001)$, DR spectra exhibit different morphologies. The DR spectra in the ML regime on $\mathrm{KCl}(001)$ ( $\alpha$ phase), allow us to assign precisely the $S_{00} \rightarrow S_{10}, S_{00} \rightarrow$ $S_{11}$ and $S_{00} \rightarrow S_{12}$ transitions. The position of the $S_{00} \rightarrow$ $S_{10}$ transition on $\mathrm{KCl}(001)$ is similar to the corresponding transition of the molecule in solvent. The vibronic replicas seen in the DR spectrum $\left(S_{00} \rightarrow S_{11}\right.$ and $\left.S_{00} \rightarrow S_{12}\right)$ coincide with the ones predicted by the fluorescence transitions by the mirror rule. Above $1 \mathrm{ML}$, with the condensation of the molecules into $\beta$-phase domains, the molecules undergo a modification of their energy levels, which is traced in the $S_{00} \rightarrow$ $S_{12}$ transition whose oscillator strength becomes prominent. Such a reversal intensity translates as a blueshift of the $\lambda_{\max }$, which is readily visible in the spectra. A more careful inspection of the spectra also reveals that on $\mathrm{KCl}(001)$, the three vibronic replicas are rigidly redshifted $(\simeq-45 \mathrm{meV})$ from 1 to $5 \mathrm{ML}$. 
With $\mathrm{NaCl}(001)$, the vibronic replicas, despite observed between 1 and $5 \mathrm{ML}$, are not as pronounced as on $\mathrm{KCl}(001)$. Nevertheless, their positions match exactly those seen on $\mathrm{KCl}(001)$ for $5 \mathrm{ML}$ too.

A thorough fit procedure of the DR spectra has allowed us to extract the intrinsic anisotropic dielectric function of the molecules in $\alpha$ and $\beta$ phases, irrespectively from the DRS formalism. The procedure relies on the combination between a proper model to account for the components of the dielectric tensor and the transfer matrix formalism. In particular, for the $\alpha$ phase, a model was developed on purpose, which explicitly accounts for the structural disorder of the phase seen in ncAFM. The fit parameters unambiguously confirm that $\alpha$ and $\beta$ phases behave optically differently. We interpret the transition between those phases as a optical transition from a monomer to an oligomer. Although the 5-ML DR spectra shapes differ between $\mathrm{KCl}(001)$ and $\mathrm{NaCl}(001)$ due to different growth modes, the results of the fits trace the presence of the same $\beta$ phase. The oligomeric state revealed in this phase yields the observed blueshift of the DR spectra, hence tracing the preliminary stages of $\mathrm{H}$ aggregation of the molecules.

\section{ACKNOWLEDGMENTS}

The authors acknowledge the financial support from the ANR PHOTONET project, grant ANR-16-JTIC-0002 of the French Agence Nationale de la Recherche, S. Clair (IM2NP, France), A. Merlen (IM2NP), T. Fritz (Jena Univ., Germany), and P. Zeppenfeld (JKU Univ., Austria) are acknowledged for helpful discussions. F. Derivaux (IM2NP) is acknowledged for technical support.

\section{APPENDIX}

The goal of this section is to give to the reader a comprehensive set of elements that are required to understand our results in a single document. We first introduce our experimental setup and detail how the DR spectra are acquired and post-processed. We then step into a theoretical part which summarizes the generic context of DRS and detail the procedure that was used to fit the DR spectra.

\section{Experimental setup}

Our DRS setup is implemented in the preparation chamber of a UHV setup consisting of two interconnected chambers (base pressure $\simeq 10^{-10}$ mbar), as shown in Fig. 15, which is approximately scaled. The light is injected and collected ex situ (atmospheric side). No optical component is installed in situ. The injection is performed through the upper right viewport, which is $45^{\circ}$ tilted with respect to the horizontal axis and reflected back by the sample towards the bottom viewport to be collected by the spectrometer. Therefore the injectioncollection angle for the light is $135^{\circ}$ and in the incidence plane of the substrate, the incidence angle of the light w.r.t. the normal axis of the substrate is $\theta_{0}=67.5^{\circ}$. Injection and collection parts are mounted on mechanical housings which allow for a fine adjustment of all parts, but which are not detailed in Fig. 15. For the readability of the figure, injection and collection parts have been displayed apart from those housings. In particular, note that the dashed arrows do not depict pathes of optical

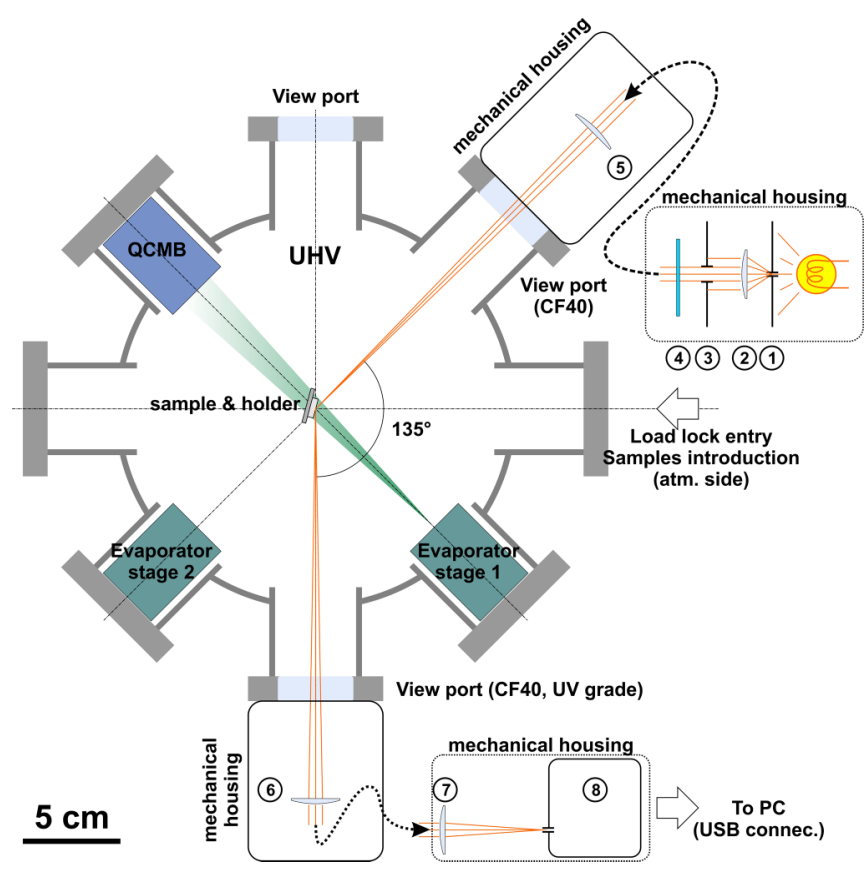

FIG. 15. Sketch of DRS setup implemented on our UHV chamber, to scale.

fibers. In order to improve the overall mechanical stability of the setup and to limit optical losses, no optical fibers are used in our setup. Therefore all optical parts are aligned to inject and collect the light properly.

The light source is a tungsten-halogen lamp (ThorLabs, SLS251), which is driven by a stabilized DC power supply (PS) (Elektro-Automatik, EA-PS 2016-050). The PS is operated at a constant power of $9 \mathrm{~W}(5.0 \mathrm{~V}, 1.8 \mathrm{~A})$. The lamp is switched on several hours prior to perform any DRS measurement in order to achieve proper emission stability (12 h typ.). Its reflectance spectrum, as measured by our spectrometer on a bare $\mathrm{KCl}(001)$ sample, is reported in Fig. 16(a). With this setup, a sensitivity of $\simeq 10^{-3}$ is reached during several hours [Figs. 16(b) and 16(c)]. The DRS experiments are performed with unpolarized light without possibility to split $s$ and $p$ polarized parts in the collected light so far. The light passes through a $1 \mathrm{~mm}$-wide pinhole (component 1 in Fig. 15) and is collimated by an achromatic doublet (component 2) with a $25 \mathrm{~mm}$ diameter and a $75 \mathrm{~mm}$ focal length (Edmund Optics, $\sharp 65-978$ ). An iris diaphragm is used to reduce the light intensity and limits the beam size to a diameter of about $7 \mathrm{~mm}$ (component 3 ). The light intensity of our lamp being maximum in the 600-900 nm range, a color balancing filter (Thorlabs, FGT165) is used, which reduces the intensity in that range and hence relatively increases the intensity in the $400-500 \mathrm{~nm}$ range (component 4). This prevents over exposure of the light to be measured by the spectrometer at $600 \mathrm{~nm}$.

The viewports are mounted on CF40 flanges and are $\simeq 170 \mathrm{~mm}$ away from the center of the UHV chamber where the sample lies. For the light injection, a regular fused quartz viewport from Caburn-MDC is used whose transmittance yields $90 \%$ and is flat in the 300-1000 $\mathrm{nm}$ range (cf. Ref. [102]). For light collection, a UV grade viewport (similar supplier) is used, whose transmittance is nearly similar and flat in 

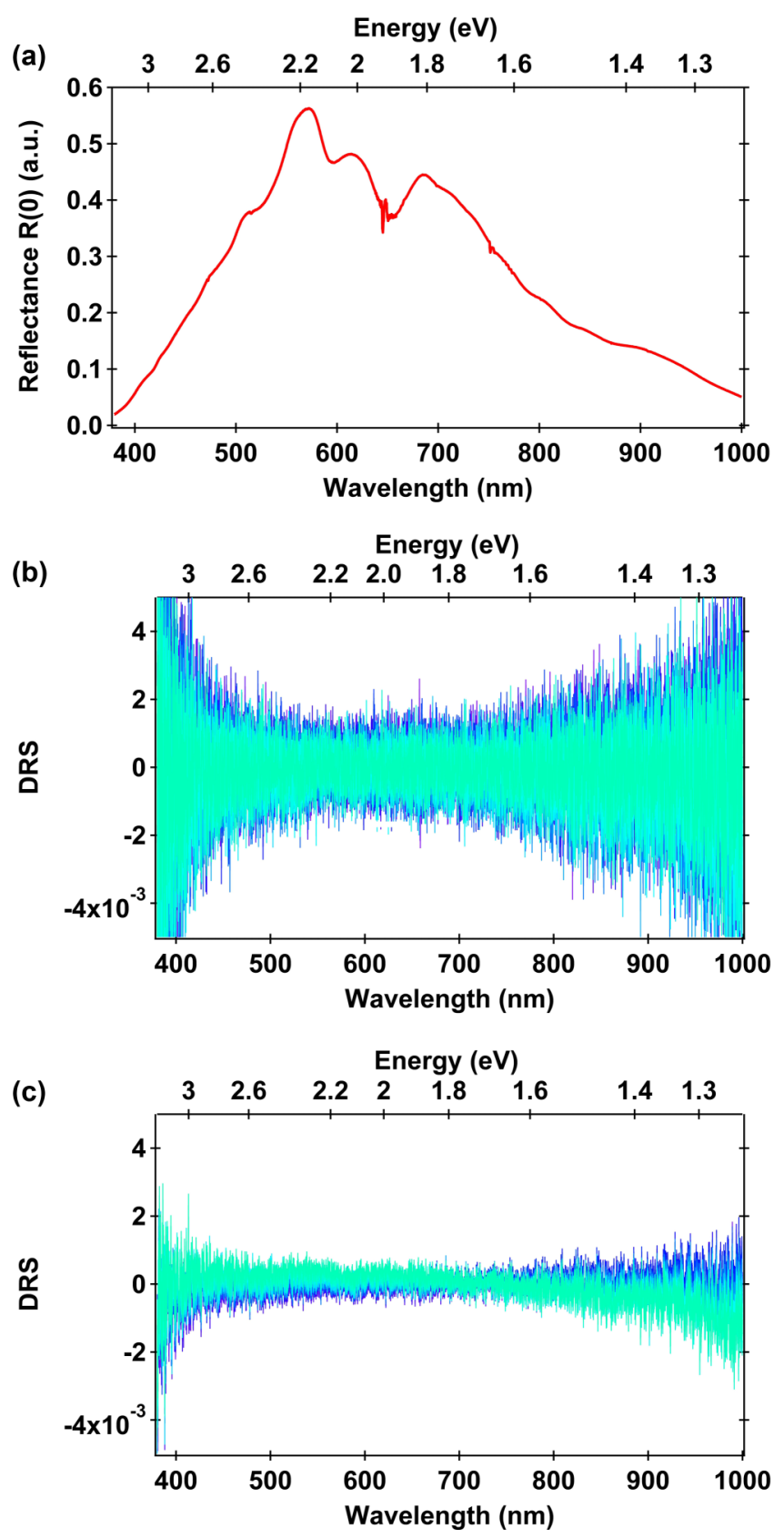

FIG. 16. (a) Typical reflectance signal measured on a bare $\mathrm{KCl}(001)$ sample used as reference for the DR spectra [ $R(0)$ signal]. (b) Illustration of the stability of the DRS measurements. The DR spectra shown here are raw data recorded over 4 hours (4100 spectra, $3.5 \mathrm{~s}$ per spectrum) on a bare $\mathrm{KCl}(001)$ sample, without any adlayer deposited. In the 450-800 $\mathrm{nm}$ interval, the fluctuations of the DRS signal are in the $\pm 210^{-3}$ range. (c) Illustration of the post-processing of the DR spectra. The raw spectra are averaged (here 100 raw spectra yield one mean spectrum), hence lowering the range of the fluctuations down to $\pm 10^{-3}$.

the 200-1000 nm range. An achromatic doublet (Thorlabs, AC254-200-A, diam. $25 \mathrm{~mm}$ ) with $200 \mathrm{~mm}$ focal length is used to focus the light on the sample (component 5). The maximum numerical aperture (N.A.) of the incident beam is $25 /(2 \times 200) \simeq 0.062$, which is equivalent to a $3.6^{\circ}$ radius light cone. Due to the iris diaphragm, the actual N.A. of the incident beam is $7 /(2 \times 200) \simeq 0.017$, which is equivalent to a $1.0^{\circ}$ radius light cone. With the optical adjustments used, we typically reach an apparent diameter of the beam light on the surface of the sample of $\simeq 2 \mathrm{~mm}$. The light reflected on the sample is collected by another AC254-200-A achromatic doublet and collimated (component 4). Finally, the light is collected into a compact CCD spectrometer (Thorlabs, CCS200, component 8 in Fig. 15) using an achromatic doublet of $25 \mathrm{~mm}$ diameter and $50 \mathrm{~mm}$ focal length (component 7). This gives a maximum N.A. at the spectrometer entrance of 0.235 , slightly larger than the specified value of 0.22 . With the typical diaphragm diameter of $7 \mathrm{~mm}$, the N.A. is 0.070 , and thus it can be considered that no light leaks out from the spectrometer entrance.

The spectrometer has a Czerny-Turner design with no moving part. The collimated light is dispersed by a blazed grating with 600 lines $/ \mathrm{mm}$ and focused on a Toshiba CCD sensor TCD1304DG. Although the spectrometer can measure in the $200-1000 \mathrm{~nm}$ range, the lower acquisition range is restricted to about $380 \mathrm{~nm}$ as the spectral component of the light source drops down to relatively small intensities for shorter wavelengths. The CCD sensor has 3648 pixels with $8 \mu \mathrm{m} \times 200 \mu \mathrm{m}$ dimensions each. It has an electronic shutter with a minimum integration time of $10 \mu$ s and typical master clock of $2 \mathrm{MHz}$. This implies that, to have an error less than $10^{-3}$ in integration time, each spectrum must be acquired within $1-10 \mathrm{~ms}$. Therefore the integration time is set to a minimum of $10 \mathrm{~ms}$ and the light intensity is reduced by the diaphragm upon needs. The CCS200 also includes an integrated circuit that reads out the value from the CCD to the USB 2.0 interface. This module performs 16 bits A/D conversion and can also subtract and normalize by a value set at the factory, giving an array of either raw or normalized [0.1, cf. Fig. 16(a)] floating point values to the computer within $5 \mathrm{~ms}$ per spectrum. Therefore our temporal limit is of $15 \mathrm{~ms}$ per spectrum.

\section{DR spectra acquisition and postprocessing}

A dedicated LabVIEW program gets the data from the spectrometer. Each processed spectrum results from the average over an arbitrary number $N$ of spectra $(N \geqslant 100)$ from the spectrometer. Thus the acquisition duration of a single spectrum lasts $\simeq 1.5 \mathrm{~s}$. This not only lowers thermal noise in the $\mathrm{CCD}$, but also reduces the amount of data to be processed, assuming that the interested phenomena are slower than this interval (see below). The averaged spectrum is then smoothed by Gaussian convolution (width $=7$ pixels, i.e., a $3.3 \mathrm{~nm}$ FWHM).

The DR spectra are then calculated after having set the reference reflectance signal $R(0)$ of the DRS measurement [cf. Fig. 16(a)]. This is done using another arbitrary $M$ number of spectra $(M \geqslant 50)$, which are averaged to define the reference spectrum. Each subsequent DR spectrum is then calculated using Eq. (1).

Owing to the sublimation rates that are typically used $(\simeq 0.3 \mathrm{ML} / \mathrm{min}$ ) as well as the regimes investigated (up to few ML, i.e., $\simeq 30$ min exposure), a DRS measurement ultimately consists of several hundreds of spectra. These are further averaged down to an arbitrary number of spectra (30 typ.). 

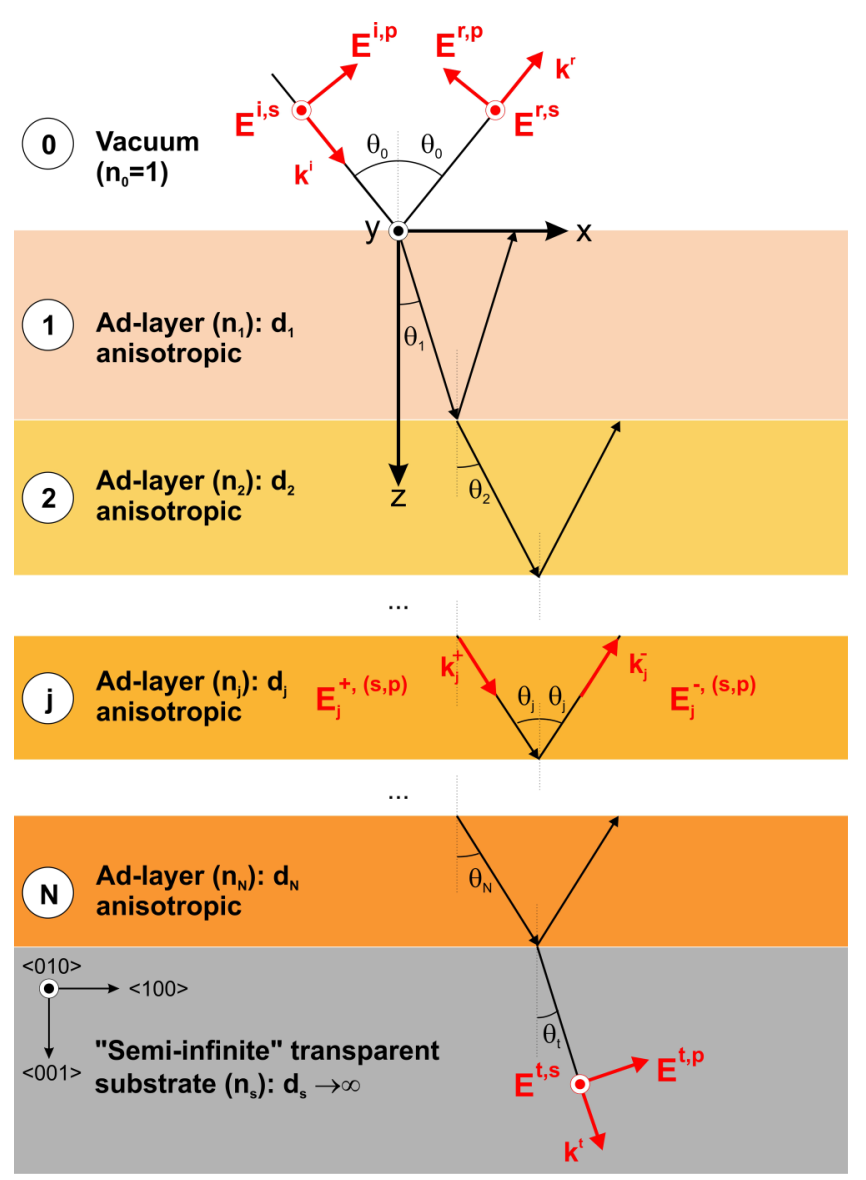

FIG. 17. Geometry of the stratified medium used to describe the DRS formalism.

At last, the DR spectra may also be drift-corrected upon needs using a linear least square fitting in a range of wavelengths where no absorption peak is present (650-800 nm typ.).

\section{Geometry of a non-magnetic stratified medium consisting of uniaxial layers with parallel optic axes}

To fit the experimental DR spectra measured on the bispyrene crystals, we consider the case of a nonmagnetic $\left(\mu_{r}=\right.$ 1) multilayered system consisting of $N$ layers with various thicknesses. The as-stratified medium is placed between two isotropic and transparent media, namely the vacuum and the bulk dielectric crystalline substrate (cf. Fig. 17). The adlayers have all thicknesses much thinner than the substrate, which can be considered as semi-infinite.

We arbitrary set the reference directions of the problem from the crystalline substrate axes, namely: $x=\langle 100\rangle, y=\langle 010\rangle$, and $z=\langle 001\rangle$. Note that the $z$ axis is referenced from the $0-1$ interface and oriented $>0$ towards the stratified medium. The incident light propagates from vacuum towards the stratified medium forming an incident angle $\theta_{0}$ w.r.t. the $z$ axis. Because $x$ and $y$ axes are equivalent in our problem (cf. below), this defines the incident place as for instance the $(x, z)$ plane.

Owing to the anisotropy of the bis-pyrene molecule and the known orientation of its dipole transition moment, it is assumed that each constitutive layer of the stratified medium behaves as an uniaxial crystal whose optic axis is oriented along the $z$ axis. This is supposed to mimic at best both, the stacking and the various orientations of the crystalline layers of bis-pyrene molecules during their growth ( $\alpha$ and $\beta$ phases) on $\mathrm{KCl}(001)$. Then, $x$ and $y$ axes are equivalent (isotropy of the problem in the $(\mathrm{x}, \mathrm{y})$ plane). Any light polarized along those axes will have an ordinary behavior (index $o$ ), whereas any other light polarization will have an extraordinary behavior (index $e$ ). Following this, the dielectric tensor of the $j^{\text {th }}$ layer writes

$$
\overline{\bar{\epsilon}}_{j}=\left(\begin{array}{ccc}
\widetilde{\epsilon}_{j}^{\perp}(\lambda) & 0 & 0 \\
0 & \widetilde{\epsilon}_{j}^{\perp}(\lambda) & 0 \\
0 & 0 & \widetilde{\epsilon}_{j}^{\|}(\lambda)
\end{array}\right),
$$

where the indexes $\perp$ and $\|$ depict the perpendicular and parallel directions to the optic axis. If optical absorption occurs within the layer $(\perp$ or $\|$ direction), the tensor components are complex (symbol $\tilde{\epsilon}$ ), real and constant otherwise.

In the following, it is convenient to use the refractive index tensor of the layers instead of the dielectric tensor: $\overline{\bar{n}}_{j}=\Re\left\{\overline{\bar{n}}_{j}\right\}+i \Im\left\{\overline{\bar{n}}_{j}\right\}=\sqrt{\overline{\bar{\epsilon}}_{j}}$, with $j=1, \ldots, N$. Note that both our conventions of complex notations for $\tilde{n}_{j}(\lambda)$ and for the electromagnetic field means that to account for an absorbing medium, one must have $\Im\left\{\tilde{n}_{j}(\lambda)\right\}<0$. For the vacuum, $\mathrm{KCl}(001)$ and $\mathrm{NaCl}(001)$, we used $n_{0}=1, n_{\mathrm{KCl}}=1.48$ and $n_{\mathrm{NaCl}}=1.53$, respectively.

\section{Models for the dielectric tensor components \\ a. Homogeneous case}

In the case where a given layer of the stratified medium is supposed to be homogeneous in the $\perp$ or $\|$ direction, then the formal expression for $\widetilde{\epsilon}_{j}^{(\perp, \|)}(\lambda)$ is given by the Lorentz oscillator model [cf. Eq. (5)]. It is the case of the $\|$ component of the 5-ML $\beta$ phase on $\mathrm{KCl}(001)\left[\widetilde{\epsilon}_{\beta, \mathrm{LOM}}^{\|}(\lambda)\right]$. Conditionally, the LOM can be combined with the effective medium theory [cf. Eq. (7)] to account for growth front effects (cf. Sec. III E2).

\section{b. Inhomogeneous case: transitions broadening}

In the $1-\mathrm{ML} \alpha \mathrm{KCl}(001)$ case, a partial structural disorder of the layer was evidenced. The homogeneous case must be revised then, as it is improper to fit the DR spectra with a good accuracy. Then, it is an inhomogeneous model for the $\perp$ component of the dielectric tensor which is to be found $\left[\widetilde{\epsilon}_{\alpha, \text { inh. }}^{\perp}(\lambda)\right]$.

For each transition, instead of a single Lorentz oscillator, i.e., a single set of wavelength, oscillator strength and lifetime, one assumes a distribution of oscillators whose oscillator strengths spread around each absorption wavelength following a Gaussian envelope [cf. Eq. (6)]. As a matter of fact, this integral-equation makes the fit impossible. Its discrete wavelength version might be used instead:

$$
\widetilde{\epsilon}_{\alpha, \text { inh. }}^{\perp}(\lambda)=\epsilon^{\infty}+\sum_{m=1}^{3} \widetilde{\epsilon}_{m}(\lambda),
$$

where $\tilde{\epsilon}_{m}(\lambda)$ stands for the model-dependent dielectric function assigned to the $m$ th transition observed in the DR spectrum (not to be confused with the dielectric function of the $m$ th layer in 
the stratified medium):

$$
\tilde{\epsilon}_{m}(\lambda)=\frac{1}{\sqrt{2 \pi} \sigma_{m}} \sum_{p} \frac{e^{-(p \delta \lambda)^{2} / 2 \sigma_{m}^{2}} f_{m} \delta \lambda}{1-\left(\frac{\lambda_{m}-p \delta \lambda}{\lambda}\right)^{2}+i \gamma_{m}\left(\frac{\lambda_{m}}{\lambda}\right)} .
$$

The summation over $p$ ranges from $-\Delta \lambda / \delta \lambda$ to $+\Delta \lambda / \delta \lambda$, $\Delta \lambda$ and $\delta \lambda$ being the range of wavelengthes over which the convolution takes place and the discrete-wavelength increment, respectively. $\sigma_{m}$ is the regular FWHM of the Gaussian function. But, even this form remains hardly tractable as it requires too much CPU resource while fitting. Thus an even stronger approximation to $\widetilde{\epsilon}_{m}(\lambda)$ is required.

Let $\tilde{\eta}_{m}(\lambda)$ be this approximated dielectric function:

$$
\tilde{\eta}_{m}(\lambda)=\Re\left\{\tilde{\eta}_{m}(\lambda)\right\}+i \Im\left\{\tilde{\eta}_{m}(\lambda)\right\} .
$$

A careful numerical analysis shows that the Gaussian broadening of a LOM function (with the FWHM of the Gaussian being of the same order as the one of the imaginary part of the LOM function) can be goodly approximated if the imaginary part of $\widetilde{\epsilon}_{m}(\lambda)$ is described out of a linear combination of a Gaussian function and the imaginary part of an equivalent LOM function. This combination, resembling a pseudo-Voigt function, gives the imaginary part of the approximated dielectric function

$$
\begin{aligned}
\Im\left\{\tilde{\eta}_{m}(\lambda)\right\}= & \Im\left\{\frac{L_{m}}{1-\left(\frac{\lambda_{m}^{\dagger}}{\lambda}\right)^{2}+i \frac{\lambda_{m}^{\dagger}}{\lambda} \gamma_{m}^{\dagger}}\right\} \\
& -G_{m} e^{-\left(\lambda-\lambda_{m}^{\dagger}\right)^{2} /\left(2 \sigma_{m}^{\dagger^{2}}\right)},
\end{aligned}
$$

where $L_{m}>0$ and $G_{m}>0$ are the LOM and Gaussian coefficients, respectively. $\lambda_{m}^{\dagger}, \gamma_{m}^{\dagger}$ and $\sigma_{m}^{\dagger}$ are the best parameters of the approximated dielectric function.

In Eq. (A3), the real part of $\widetilde{\epsilon}_{m}(\lambda)$ can reasonably be approximated with the real part of a properly weighted LOM function, thus

$$
\mathfrak{R}\left\{\widetilde{\eta}_{m}(\lambda)\right\}=\mathfrak{R}\left\{\frac{L_{m}+G_{m} \gamma_{m}^{\dagger}}{1-\left(\frac{\lambda_{m}^{\dagger}}{\lambda}\right)^{2}+i \frac{\lambda_{m}^{\dagger}}{\lambda} \gamma_{m}^{\dagger}}\right\} .
$$

Now, to use Eq. (A4) together with Eqs. (A5) and (A6) in the fitting process, we also force the Gaussian and Lorentz distributions to have similar widthes, namely, $\lambda_{m}^{\dagger} \gamma_{m}^{\dagger}=2 \sqrt{2 \ln (2)} \sigma_{m}^{\dagger}$. For a $m$ th transition, this ultimately yields a four-parameter dielectric function $\left(\lambda_{m}^{\dagger}, \gamma_{m}^{\dagger}, L_{m}\right.$, and $\left.G_{m}\right)$ to be injected in the DRS functional [cf. next section, Eq. (A11)].

Once $\lambda_{m}^{\dagger}, \gamma_{m}^{\dagger}, L_{m}$, and $G_{m}$ are fitted, hence fully defining Eq. (A4), the actual oscillator strength $f_{m}$, exact peak position $\lambda_{m}$ and $\gamma_{m}$ parameter of each transition (cf. Table III) are obtained by fitting the imaginary part of $\widetilde{\eta}_{m}(\lambda)$ [cf. Eq. (A4)] with the imaginary part of $\widetilde{\epsilon}_{m}(\lambda)$ [cf. Eq. (A3)], while ensuring that the results neither depend on $\Delta \lambda$, nor on $\delta \lambda$.

\section{DRS formalism and fitting functional}

In the context of the geometry of Fig. 17, the $s$ - (perpendicular to the plane of incidence) and $p$-polarized (parallel to the plane of incidence) components (electric transverse and magnetic transverse waves, respectively) of the electric part of the incident $(i)$, reflected $(r)$ and transmitted $(t)$ electromagnetic field, $\mathbf{E}^{\dagger}=\mathbf{E}^{\dagger, s}+\mathbf{E}^{\dagger, p}$, (with $\dagger=i, r$, or $t$ ) write

$$
\begin{aligned}
& \mathbf{E}^{\dagger, s}=E^{\dagger, y} \mathbf{e}_{y} e^{i\left[\omega t-k^{\dagger, x} x-k^{\dagger, z} z\right]} \\
& \mathbf{E}^{\dagger, p}=\left\{E^{\dagger, x} \mathbf{e}_{x}+E^{\dagger, z} \mathbf{e}_{z}\right\} e^{i\left[\omega t-k^{\dagger, x} x-k^{\dagger, z} z\right]}
\end{aligned}
$$

with $\mathbf{k}^{\dagger}=k^{\dagger, x} \mathbf{e}_{x}+k^{\dagger, y} \mathbf{e}_{y}+k^{\dagger, z} \mathbf{e}_{z}$, the wave vector of the light.

Generically, the reflection coefficient due to the molecular layers of equivalent thickness $d$ writes

$$
r^{(s, p)}(d)=\frac{\left\|\mathbf{E}^{r,(s, p)}(d)\right\|}{\left\|\mathbf{E}^{i,(s, p)}\right\|} .
$$

The corresponding reflectance is thus defined as

$$
R^{(s, p)}(d)=\left|r^{(s, p)}(d)\right|^{2},
$$

Hence the $s$ and $p$ components of the DRS signal:

$$
\operatorname{DRS}^{(s, p)}(d)=\frac{R^{(s, p)}(d)-R^{(s, p)}(0)}{R^{(s, p)}(0)} .
$$

The case of unpolarized light $(u-)$, i.e., our actual measured DRS signal, is derived as the superposition of $s$ and $p$ components, $R^{u}(d)=\left[R^{s}(d)+R^{p}(d)\right] / 2$,

$$
\begin{aligned}
\operatorname{DRS}^{u}(d) & =\frac{R^{u}(d)-R^{u}(0)}{R^{u}(0)} \\
& =\frac{R^{s}(0) \operatorname{DRS}^{s}(d)+R^{p}(0) \operatorname{DRS}^{p}(d)}{R^{s}(0)+R^{p}(0)} .
\end{aligned}
$$

After derivation of $\mathbf{E}_{r}^{(s, p)}(d)$ as a function of the retained dielectric function model for each layer of the stratified medium (cf. next section), Eq. (A11) depicts the fitting functional of the DR spectra. The fitting process is performed by a Wolfram MATHEMATICA ${ }^{\circledR}$ program based on a Levenberg-Marquardt algorithm. The otherwise constant parameters are the refractive index of vacuum, $\mathrm{KCl}$ and $\mathrm{NaCl}$ (yet given), as well as the light incidence angle, $\theta_{0}=65^{\circ}$.

\section{The transfer matrix method (TMM)}

$\mathbf{E}_{r}^{(s, p)}(d)$ can be derived out of the transfer matrix method [83] (TMM), which is based on the Maxwell's equations and the continuity conditions of the in-plane electric and magnetic fields components at each interface of the stratified medium. Note that the $s$ and $p$ components splitting of the fields remains valid because the stratified medium considered here consists of uniaxial layers whose optic axes are all parallel and contained within the incidence plane [83] (i.e., perpendicular to the scomponent, cf. Fig. 17).

We start from the wave vector of the light, whose incident (" $i,+$ ") and reflected (" $r,-$ ") components within a $j$ th layer can be written, using the Snell-Descartes' equation, as

$$
\mathbf{k}_{j}^{(i, r)}=k_{j}^{(i, r), x} \mathbf{e}_{x}+k_{j}^{(+,-), z} \mathbf{e}_{z},
$$

where

$$
\begin{aligned}
k_{j}^{(i, r), x} & =\frac{2 \pi}{\lambda} n_{0} \sin \left(\theta_{0}\right) \\
k_{j}^{(+,-), z} & = \pm \frac{2 \pi}{\lambda} \tilde{n}_{j}(\lambda) \cos \left(\theta_{j}\right) .
\end{aligned}
$$

This allows for writing any component of the incident or reflected electric field within the $j$ th layer. For instance, the 
component of the incident or reflected $s$ component, writes with the,+- notations

$$
E_{j}^{(+,-), s}=E_{j}^{(+,-), y} e^{i\left[\omega t-k^{(+,-), x} x-k^{(+,-), z} z\right]} .
$$

As mentioned, the Maxwell's equations and the continuity conditions of the in-plane fields components at each interface allows us to derive the $s$ and $p$ components of the electromagnetic field inside each layer of the stratified medium. For an interface between the $(j-1)$ th and the $j$ th layers, it can be shown that the in-plane $E_{j}^{(+,-), y}$ ( $s$ polarization) and $E_{j}^{(+,-), x}$ ( $p$ polarization) components of the electric field write similarly, namely, with simplified notations (indexes $x$ or $y$ omitted)

$$
\begin{gathered}
E_{(j-1)}^{+}+E_{(j-1)}^{-}=E_{j}^{+} e^{i \beta_{j}}+E_{j}^{-} e^{-i \beta_{j}} \\
\alpha_{(j-1)}\left(E_{(j-1)}^{+}-E_{(j-1)}^{-}\right)=\alpha_{j}\left(E_{j}^{+} e^{i \beta_{j}}-E_{j}^{-} e^{-i \beta_{j}}\right),
\end{gathered}
$$

where $\alpha_{j}$ and $\beta_{j}$ are $s$ - or $p$-dependent parameters and given hereafter. In the case of the last interface ( $N$ th layer/substrate), the former set of boundary equations writes

$$
\begin{gathered}
E_{N}^{+}+E_{N}^{-}=E^{t} \\
\alpha_{N}\left(E_{N}^{+}-E_{N}^{-}\right)=\alpha_{s} E^{t} .
\end{gathered}
$$

Thus it is possible to recursively derive a matrix relationship between the incident, reflected and transmitted electric field for both in-plane components of $s$ - and $p$ polarizations:

$$
\left(\begin{array}{l}
E^{i,(x, y)} \\
E^{r,(x, y)}
\end{array}\right)=\frac{1}{2} \prod_{j=1}^{N} T_{j}\left(\begin{array}{l}
{\left[1+\frac{\alpha_{s}}{\alpha_{N}}\right] E^{t,(x, y)}} \\
{\left[1-\frac{\alpha_{s}}{\alpha_{N}}\right] E^{t,(x, y)}}
\end{array}\right),
$$

where $T_{j}$ is a matrix given by

$$
T_{j}=\frac{1}{2}\left(\begin{array}{l}
{\left[1+\frac{\alpha_{j}}{\alpha_{(j-1)}}\right] e^{i \beta_{j}}\left[1-\frac{\alpha_{j}}{\alpha_{(j-1)}}\right] e^{-i \beta_{j}}} \\
{\left[1-\frac{\alpha_{j}}{\alpha_{(j-1)}}\right] e^{i \beta_{j}}\left[1+\frac{\alpha_{j}}{\alpha_{(j-1)}}\right] e^{-i \beta_{j}}}
\end{array}\right) .
$$

From Eq. (A17) by removing $E^{t,(x, y)}$, it is straightforward to derive an in-plane reflection coefficient

$$
r^{(x, y)}(d)=\frac{\left|E^{r,(x, y)}(d)\right|}{\left|E^{i,(x, y)}\right|} .
$$

(1) $s$-polarization case. Here $E^{(+,-), s}=E^{(+,-), y}$. The electric field is perpendicular to the optic axis. Thus it behaves "ordinarily" (index " $o$ "). Then

$$
\begin{aligned}
& \alpha_{j}=\alpha_{j}^{o}=\widetilde{n}_{j}^{\perp} \cos \left(\theta_{j}^{o}\right) \overbrace{=}^{\text {Snell-Descartes }} \sqrt{\widetilde{n}_{j}^{\perp^{2}}-n_{0}^{2} \sin ^{2}\left(\theta_{0}\right)} \\
& \beta_{j}=\beta_{j}^{o}=\frac{2 \pi d_{j}}{\lambda} \alpha_{j}^{o} .
\end{aligned}
$$

In this polarization, the in-plane reflection coefficient [cf. Eq. (A19)] matches the reflection coefficient [cf. Eq. (A8)]: $r^{y}(d)=r^{s}(d)$.

(2) $p$-polarization case. Here $\mathbf{E}^{(+,-), p}=E^{(+,-), x} \mathbf{e}_{x}+$ $E^{(+,-), z} \mathbf{e}_{z}$. The electric field is now in the $(z, x)$ plane (not perpendicular to the optic axis) and behaves extraordinarily (index " $e$ "). It is found

$$
\begin{aligned}
& \alpha_{j}=\alpha_{j}^{e}=\frac{\tilde{n}_{j}^{\perp^{2}}}{\tilde{n}_{j} \cos \left(\theta_{j}^{e}\right)} \\
& \beta_{j}=\beta_{j}^{e}=\frac{2 \pi d_{j}}{\lambda} \tilde{n}_{j} \cos \left(\theta_{j}^{e}\right) .
\end{aligned}
$$

The Snell-Descartes' equation combined with the index ellipsoid equation which connects the extraordinary propagation direction to the $\perp$ and $\|$ indexes, namely,

$$
\frac{\cos ^{2}\left(\theta_{j}^{e}\right)}{\widetilde{n}_{j}^{\perp^{2}}}+\frac{\sin ^{2}\left(\theta_{j}^{e}\right)}{\widetilde{n}_{j}^{\|^{2}}}=\frac{1}{\widetilde{n}_{j}^{2}},
$$

ultimately yields to

$$
\begin{aligned}
\alpha_{j}^{e} & =\frac{\widetilde{n}_{j}^{\perp} \widetilde{n}_{j}^{\|}}{\sqrt{\widetilde{n}_{j}^{\|^{2}}-n_{0}^{2} \sin ^{2}\left(\theta_{0}\right)}} \\
\beta_{j}^{e} & =\frac{2 \pi d_{j}}{\lambda} \frac{\widetilde{n}_{j}^{\perp}}{\alpha_{j}^{e}} .
\end{aligned}
$$

Here, due to the reflection law (equality between incident and reflection angles, cf. Fig. 17), $\left|E^{(i, r), x}\right|=\left\|\mathbf{E}^{(i, r), p}\right\| \times$ $\left|\cos \left(\theta_{0}\right)\right|$, therefore the in-plane reflection coefficient $r^{x}(d)=$ $r^{p}(d)$ [cf. Eqs. (A8) and (A19)].

(3) DRS functional. The combination between Eqs. (A23), (A20), (A19), and (A1) and the retained model for the dielectric tensor components [Eq. (5) for the LOM, Eq. (7) for the EMT+LOM, and Eqs. (A4)-(A6) for the inhomogeneous model] allows for the derivation of the reflectance [cf. Eq. (A9)] and hence, the unpolarized DRS functional [cf. Eq. (A11)].

\section{Application of the TMM}

We illustrate the TMM formalism to simulate DR spectra applied to two cases. The first deals with the redshift observed in the 5-ML $\beta / \alpha \mathrm{KCl}(001)$ case. The second deals with the influence of the roughness of the top-most layer on the resulting DR spectra.

\section{a. On the origin of the redshift of the 5-ML $\beta / \alpha$ case}

It is argued in the text that the redshift of the peaks in the 5-ML $\beta / \alpha \mathrm{KCl}(001)$ case stems from the $\beta$-phase optical response, which gets strengthened as the $\beta$-phase layers grow on top of the first $\alpha$-phase ML. Thus the measured DR spectrum on $\mathrm{KCl}(001)$ results from a mixing of the optical responses of the $\alpha$ phase and the growing $\beta$ phase. This trend was simulated by the TMM in the case of a system consisting of three interfaces [cf. Fig. 18(a)]: vacuum/top-most $\beta$-phase layer, $\beta / \alpha$, and $\alpha /$ substrate. The dielectric functions of the two anisotropic layers are those of the corresponding phase, as derived from the fits of the experimental DR spectra. The thickness of the $\alpha$ phase is kept constant $\left(d_{\alpha}=1.1 \mathrm{~nm}\right)$ whereas the thickness of the $\beta$-phase layer is gradually increased: $d_{\beta}=0, \ldots, 10 \mathrm{~nm}$, thus mimicking the $\beta$-phase layer-by-layer growth mode.

The simulated DR spectra are reported in Fig. 18(b). They are in excellent agreement with the experimental trends, with 
(a)
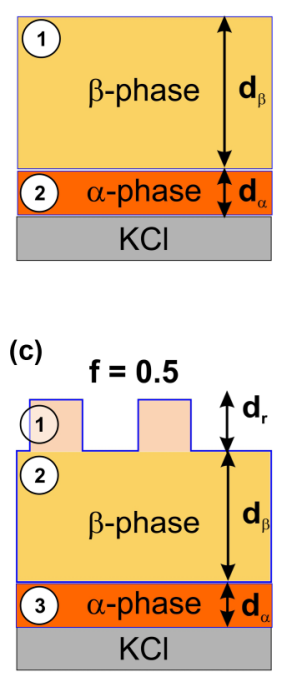

(b) Energy (eV)

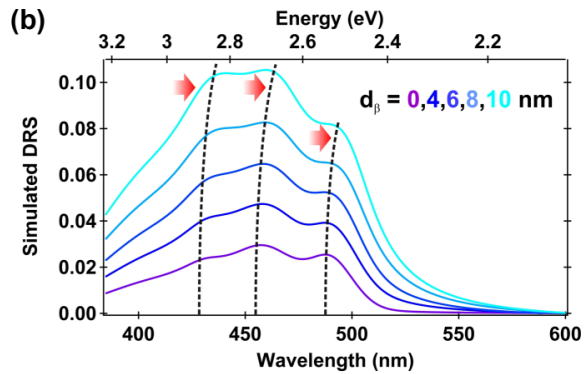

(d)

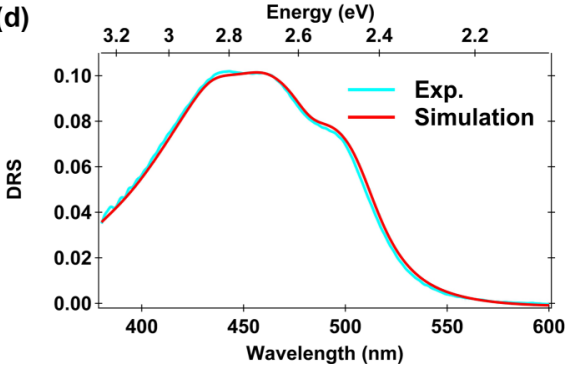

FIG. 18. [(a) and (b)] Sketch of the global composite system for $\alpha$ and $\beta$ phases overlaid and simulated DR spectra, respectively. [(c) and (d)] Sketch of the global composite system for the study of the roughness effect and simulated DR spectrum, respectively.

a moderate, $40 \mathrm{meV}$, redshift of the three transitions of the vibronic progression, which validates our guess.

\section{b. Investigation of the influence of top-most layer roughness on the DR spectra}

To estimate whether the roughness of the topmost layer has an influence on the resulting DR spectra, we have considered the global composite system shown in Fig. 18(c) consisting of three layers and hence, of four interfaces. Layer 3 has the $\alpha$ phase characteristics. Layer 2 has the dielectric function of the $\beta$ phase and its thickness is $d_{\beta}=8 \mathrm{~nm}$. The topmost layer (layer 1) is rough. It is described in the EMT approach considering a $50 \%$ filling rate and spherical islands $[f=0.5$ and $v=1 / 3$, respectively, in Eq. (7)]. In order to amplify the roughness effect, the height of the islands was defined as twice the one of a single $\beta$-phase layer, thus $d_{r}=4 \mathrm{~nm}$.

The simulated DR spectra is reported in Fig. 18(d) and overlaid on the experimental 5-ML $\beta / \alpha$ one. It can be seen that the shape of the simulated DR spectrum is not affected by the roughness of the top-most layer, despite its large magnitude. We therefore conclude that the DR spectra fits of the 5-ML $\beta / \alpha$ $\mathrm{KCl}(001)$ case considering flat interfaces is not a too severe assumption.
[1] K. Sato, K. Shizu, K. Yoshimura, A. Kawada, H. Miyazaki, and C. Adachi, Phys. Rev. Lett. 110, 247401 (2013).

[2] S. Reineke, M. Thomschke, B. Lüssem, and K. Leo, Rev. Mod. Phys. 85, 1245 (2013).

[3] K. Walzer, B. Maennig, M. Pfeiffer, and K. Leo, Chem. Rev. 107, 1233 (2007).

[4] T. Drori, C.-X. Sheng, A. Ndobe, S. Singh, J. Holt, and Z. V. Vardeny, Phys. Rev. Lett. 101, 037401 (2008).

[5] A. Mishra and P. Bäuerle, Angew. Chem. Int. Ed. 51, 2020 (2012).

[6] K. Sakai, Y. Okada, S. Kitaoka, J. Tsurumi, Y. Ohishi, A. Fujiwara, K. Takimiya, and J. Takeya, Phys. Rev. Lett. 110, 096603 (2013).

[7] M. Akai-Kasaya, Y. Okuaki, S. Nagano, T. Mitani, and Y. Kuwahara, Phys. Rev. Lett. 115, 196801 (2015).

[8] F. Dinelli, M. Murgia, P. Levy, M. Cavallini, F. Biscarini, and D. M. de Leeuw, Phys. Rev. Lett. 92, 116802 (2004).

[9] E. Menard, M. A. Meitl, Y. Sun, J.-U. Park, D. J.-L. Shir, Y.-S. Nam, S. Jeon, and J. A. Rogers, Chem. Rev. 107, 1117 (2007).

[10] A. Mishra, C.-Q. Ma, and P. Baüerle, Chem. Rev. 109, 1141 (2009).

[11] A. Shehu, S. D. Quiroga, P. D’Angelo, C. Albonetti, F. Borgatti, M. Murgia, A. Scorzoni, P. Stoliar, and F. Biscarini, Phys. Rev. Lett. 104, 246602 (2010).

[12] Y. Zhang, J. Qiao, S. Gao, F. Hu, D. He, B. Wu, Z. Yang, B. Xu, Y. Li, Y. Shi, W. Ji, P. Wang, X. Wang, M. Xiao, H. Xu, J. B. Xu, and X. Wang, Phys. Rev. Lett. 116, 016602 (2016).

[13] M. Pope and C. E. Swenberg, Electronic Processes in Organic Crystals and Polymers, 2nd ed. (Oxford University Press, New York, 1999).

[14] N. Koch, Chem. Phys. Chem. 8, 1438 (2007).

[15] M. Kozlik, S. Paulke, M. Gruenewald, R. Forker, and T. Fritz, Org. Electron. 13, 3291 (2012).
[16] A. Massé, R. Coehoorn, and P. A. Bobbert, Phys. Rev. Lett. 113, 116604 (2014).

[17] T. Hosokai et al., J. Phys. Chem. C 119, 29027 (2015).

[18] X. Qiu, G. Nazin, and W. Ho, Science 299, 542 (2003).

[19] R. Pawlak, L. Nony, F. Bocquet, V. Oison, M. Sassi, J.-M. Debierre, C. Loppacher, and L. Porte, J. Phys. Chem. C 114, 9290 (2010).

[20] S. Kawai, R. Pawlak, T. Glatzel, and E. Meyer, Phys. Rev. B 84, 085429 (2011).

[21] C. Barth, M. Gingras, A. S. Foster, A. Gulans, G. Félix, T. Hynninen, R. Peresutti, and C. R. Henry, Adv. Mater. 24, 3228 (2012).

[22] F. Bocquet, L. Nony, S. C. B.Mannsfeld, V. Oison, R. Pawlak, L. Porte, and C. Loppacher, Phys. Rev. Lett. 108, 206103 (2012).

[23] A. Amrous et al., Adv. Mater. Interfaces 1, 1400414 (2014).

[24] L. Gross, F. Mohn, N. Moll, and G. Meyer, Science 325, 1110 (2009).

[25] L. Gross, F. Mohn, N. Moll, G. Meyer, R. Ebel, W. M. AbdelMageed, and M. Jaspars, Nat. Chem. 2, 821 (2010).

[26] F. Mohn, L. Gross, N. Moll, and G. Meyer, Nat. Nanotechnol. 7, 227 (2012).

[27] D. de Oteyza et al., Science 340, 1434 (2013).

[28] A. M. Sweetman, S. P. Jarvis, H. Sang, I. Lekkas, P. Rahe, Yu. Wang, J. Wang, N. R. Champness, L. Kantorovich, and P. Moriarty, Nat. Commun. 5, 3931 (2014).

[29] S. Kawai et al., Nat. Commun. 7, 12711 (2016).

[30] J. McIntyre and D. Aspnes, Surf. Sci. 24, 417 (1971).

[31] D. E. Aspnes, J. Vac. Sci. Technol., B 3, 1498 (1985).

[32] H. Proehl, R. Nitsche, Th. Dienel, K. Leo, and T. Fritz, Phys. Rev. B 71, 165207 (2005).

[33] R. Forker, M. Gruenewald, and T. Fritz, Annu. Rep. Prog. Chem., Sect. C: Phys. Chem. 108, 34 (2012). 
[34] A. Facchetti, Chem. Mater. 23, 733 (2011).

[35] T. Figueira-Duarte and K. Müllen, Chem. Rev. 111, 7260 (2011).

[36] J. Gaberle, D. Z. Gao, A. L. Shluger, A. Amrous, F. Bocquet, L. Nony, F. Para, Ch. Loppacher, S. Lamare, and F. Cherioux, J. Phys. Chem. C 121, 4393 (2017).

[37] T. Lelaidier, T. Lünskens, A. von Weber, T. Leoni, A. Ranguis, A. D'Aléo, F. Fages, A. Kartouzian, C. Becker, and U. Heiz, Phys. Chem. Chem. Phys. 18, 5299 (2016).

[38] T. Lelaidier, T. Leoni, A. Ranguis, A. D’Aléo, F. Fages, and C. Becker, J. Phys. Chem. C 121, 7214 (2017).

[39] F. J. Giessibl and H. Bielefeldt, Phys. Rev. B 61, 9968 (2000).

[40] I. Horcas, R. Fernández, J. M. Gómez-Rodríguez, J. Colchero, J. Gómez-Herrero, and A. M. Baro, Rev. Sci. Instrum. 78, 013705 (2007).

[41] Y. Borensztein, G. Prévot, and L. Masson, Phys. Rev. B 89, 245410 (2014).

[42] A. Navarro-Quezada, M. Aiglinger, E. Ghanbari, Th. Wagner, and P. Zeppenfeld, Rev. Sci. Instrum. 86, 113108 (2015).

[43] H. Zaglmayr, C. Hu, L. Sun, and P. Zeppenfeld, Meas. Sci. Technol. 25, 115603 (2014).

[44] M. Roy and Y. Borensztein, Surf. Sci. 331-333, 453 (1995).

[45] C. Beitia and Y. Borensztein, Phys. Status Solidi A 175, 39 (1999).

[46] Y. Borensztein, O. Pluchery, and N. Witkowski, Phys. Rev. Lett. 95, 117402 (2005).

[47] K. Gaál-Nagy, A. Incze, G. Onida, Y. Borensztein, N. Witkowski, O. Pluchery, F. Fuchs, F. Bechstedt, and R. Del Sole, Phys. Rev. B 79, 045312 (2009).

[48] V. L. Berkovits, N. Witkowski, Y. Borensztein, and D. Paget, Phys. Rev. B 63, 121314(R) (2001).

[49] F. Vidal, O. Pluchery, N. Witkowski, V. Garcia, M. Marangolo, V. H. Etgens, and Y. Borensztein, Phys. Rev. B 74, 115330 (2006).

[50] H. Proehl, T. Dienel, R. Nitsche, and T. Fritz, Phys. Rev. Lett. 93, 097403 (2004).

[51] T. Dienel, C. Loppacher, S. C. B. Mannsfeld, R. Forker, and T. Fritz, Adv. Mater. 20, 959 (2008).

[52] R. Forker, T. Dienel, A. Krause, M. Gruenewald, M. Meissner, T. Kirchhuebel, O. Groning, and T. Fritz, Phys. Rev. B 93, 165426 (2016).

[53] R. Coustel, Y. Borensztein, O. Pluchery, and N. Witkowski, Phys. Rev. Lett. 111, 096103 (2013).

[54] T. Dienel, R. Forker, K. Leo, and T. Fritz, J. Phys. Chem. C Lett. 111, 14593 (2007).

[55] S. Boudet, I. Bidermane, E. Lacaze, B. Gallas, M. Bouvet, J. Brunet, A. Pauly, Y. Borensztein, and N. Witkowski, Phys. Rev. B 86, 115413 (2012).

[56] U. Heinemeyer, K. Broch, A. Hinderhofer, M. Kytka, R. Scholz, A. Gerlach, and F. Schreiber, Phys. Rev. Lett. 104, 257401 (2010).

[57] L. Zhang, X. Fu, C. G. Hu, Y. Yao, Z. Y. Xu, X. T. Hu, M. Hohage, P. Zeppenfeld, and L. D. Sun, Phys. Rev. B 93, 075443 (2016).

[58] L. Sun, S. Berkebile, G. Weidlinger, M. Denk, R. Denk, M. Hohage, G. Koller, F. P. Netzer, M. G. Ramseyb, and P. Zeppenfeld, Phys. Chem. Chem. Phys. 14, 13651 (2012).

[59] Y. Borensztein, Surf. Rev. Lett. 07, 399 (2000).

[60] R. Forker and T. Fritz, Phys. Chem. Chem. Phys. 11, 2142 (2009).
[61] E. Nichols and E. Merritt, Phys. Rev. (Series I) 31, 376 (1910).

[62] I. B. Berlman, Handbook of Fluorescence Spectra of Aromatic Molecules, 2nd ed. (Academic, New York, 1971).

[63] E. Allwright, D. M. Berg, R. Djemour, M. Steichen, P. J. Dale, and N. Robertson, J. Mater. Chem. C 2, 7232 (2014).

[64] C. Byron and T. Werner, J. Chem. Educ. 68, 433 (1991).

[65] G. Heimel, M. Daghofer, J. Gierschner, E. J. W. List, A. C. Grimsdale, K. Müllen, D. Beljonne, J.-L. Brédas, and E. Zojer, J. Chem. Phys. 122, 054501 (2005).

[66] I. Tinoco, J. Am. Chem. Soc. 82, 4785 (1960).

[67] W. Rhodes, J. Am. Chem. Soc. 83, 3609 (1961).

[68] H. Devoe, J. Chem. Phys. 41, 393 (1964).

[69] V. Agranovich and G. Bassani, in Thin Films and Nanostructures: Electronic Excitations in Organic Based Nanostructures (Elsevier Academic Press, New York, 2003), Vol. 31.

[70] F. Spano, Acc. Chem. Res. 43, 429 (2010).

[71] F. Spano and C. Silva, Annu. Rev. Phys. Chem. 65, 477 (2014).

[72] C. Barth and C. R. Henry, Phys. Rev. Lett. 98, 136804 (2007)

[73] A. Hinaut, A. Pujol, F. Chaumeton, D. Martrou, A. Gourdon, and S. Gauthier, Beilstein J. Nanotechnol. 3, 221 (2012).

[74] This is readily seen in a molecular dynamic simulation of a molecule with similar side chains adsorbed on $\mathrm{KCl}(001)$. See the supporting information in Ref. [36].

[75] R. Forker, M. Meissner, and T. Fritz, Soft Matter 13, 1748 (2017)

[76] G. Hlawacek, P. Puschnig, P. Frank, A. Winkler, C. AmbroschDraxl, and C. Teichert, Science 321, 108 (2008).

[77] S. Leroy-Lhez, M. Allain, J. Oberlé, and F. Fages, New J. Chem. 31, 1013 (2007).

[78] D. Aspnes, J. Opt. Soc. Am. 70, 1275 (1980).

[79] M. Alonso, M. Garriga, F. Alsina, and S. Piñol, Appl. Phys. Lett. 67, 596 (1995).

[80] J.-F. Nye, in Physical Properties of Crystals (Clarendon Press, Oxford, 1957).

[81] M. Alonso and M. Garriga, Thin Solid Films 455-456, 124 (2004).

[82] N. A. Nemkovich, A. N. Rubinov, and I. T. Tomin, in Topics in Fluorescence Spectroscopy, Vol. 2: Principles. Inhomogeneous Broadening of Electron Spectra of Dye Molecules, edited by J. Lakowicz (Plenum Press, New York, 1991), pp. 367-428.

[83] R. Azzam and N. Bashara, Ellipsometry and Polarized Ligth (North Holland, Amsterdam, 1977).

[84] J. Lekner, in Theory of Reflection of Electromagnetic and Particle Waves (Martinus Nijhoff Publishers, Dordrecht, 1987).

[85] B. J. C. Maxwell Garnet, Philos. Trans. R. Soc., A 203, 385 (1904).

[86] V. Markel, J. Opt. Soc. Am. A 33, 1244 (2016).

[87] O. Levy and D. Stroud, Phys. Rev. B 56, 8035 (1997).

[88] D. E. Aspnes, J. B. Theeten, and F. Hottier, Phys. Rev. B 20, 3292 (1979).

[89] M. Scheller, C. Jansen, and M. Koch, Applications of effective medium theories in the terahertz regime, in Recent Optical and Photonic Technologies, edited by K. Y. Kim (InTech, Rijeka, Croatia, 2010).

[90] S. Tang, B. Zhu, M. Jia, Q. He, S. Sun, Y. Mei, and L. Zhou, Phys. Rev. B 91, 174201 (2015).

[91] R. Petersen, T. G. Pedersen, M. N. Gjerding, and K. S. Thygesen, Phys. Rev. B 94, 035128 (2016). 
[92] M. Carlberg, F. Pourcin, O. Margeat, J. Le Rouzo, G. Berginc, R.-M. Sauvage, J. Ackermann, and L. Escoubas, Opt. Mater. Express 7, 4241 (2017).

[93] M. Kasha, Radiation Research 20, 55 (1963).

[94] M. Kasha, H. Rawls, and M. A. El-Bayoumi, Pure Appl. Chem. 11, 371 (1965).

[95] A. Davydov, in The Theory of Molecular Excitons (Springer, New York, 1971).
[96] J. Briggs and A. Herzenberg, Mol. Phys. 21, 865 (1971).

[97] A. Eisfeld and J. Briggs, Chem. Phys. 281, 61 (2002).

[98] A. Eisfeld and J. Briggs, Chem. Phys. 324, 376 (2006).

[99] F. Spano, Chem. Phys. 325, 22 (2006).

[100] A. Davydov, Ukr. J. Phys. (Special Issue) 53, 65 (2008).

[101] F. F. So and S. R. Forres, Phys. Rev. Lett. 66, 2649 (1991).

[102] https://www.mdcvacuum.com 